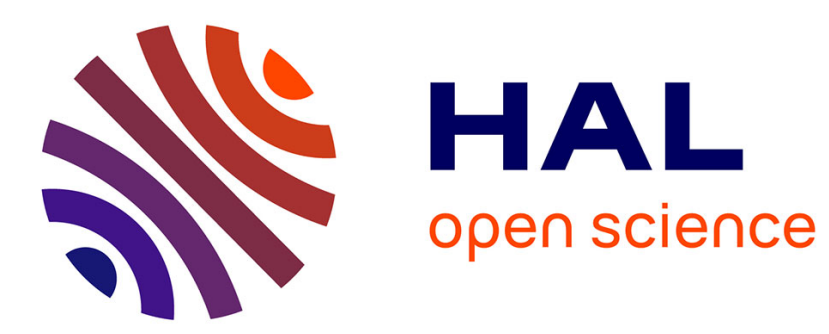

\title{
Set-valued sliding-mode control of uncertain linear systems: continuous and discrete-time analysis
}

Felix Miranda-Villatoro, Bernard Brogliato, Fernando Castaños

\section{To cite this version:}

Felix Miranda-Villatoro, Bernard Brogliato, Fernando Castaños. Set-valued sliding-mode control of uncertain linear systems: continuous and discrete-time analysis. SIAM Journal on Control and Optimization, 2018, 56 (3), pp.1756-1793. 10.1137/16M1077362 . hal-01317948

\section{HAL Id: hal-01317948 \\ https://hal.inria.fr/hal-01317948}

Submitted on 19 May 2016

HAL is a multi-disciplinary open access archive for the deposit and dissemination of scientific research documents, whether they are published or not. The documents may come from teaching and research institutions in France or abroad, or from public or private research centers.
L'archive ouverte pluridisciplinaire HAL, est destinée au dépôt et à la diffusion de documents scientifiques de niveau recherche, publiés ou non, émanant des établissements d'enseignement et de recherche français ou étrangers, des laboratoires publics ou privés. 


\title{
Set-valued sliding-mode control of uncertain linear systems: continuous and discrete-time analysis
}

\author{
Félix A. Miranda-Villatoro ${ }^{\dagger}$, Bernard Brogliato ${ }^{\ddagger}$, and Fernando Castaños ${ }^{\dagger}$ \\ †Automatic Control Department, CINVESTAV-IPN, 2508 av. Instituto Politécnico \\ Nacional, 07360, Mexico City, Mexico. \\ ¥INRIA Grenoble Rhône-Alpes, University of Grenoble-Alpes, Inovalleé, 655 av. de \\ l'Europe, 38334, Saint-Ismier, France.
}

May 18, 2016

\begin{abstract}
In this paper we study the closed-loop dynamics of linear time-invariant systems with feedback control laws that are described by set-valued maximal monotone maps. The class of systems considered in this work is subject to both, unknown exogenous disturbances and parameter uncertainty. It is shown how the design of conventional sliding mode controllers can be achieved using maximal monotone operators (which include the set-valued signum function). Two cases are analyzed: continuous-time and discrete-time controllers. In both cases well-posedness together with stability results are presented. In discrete time we show how the implicit scheme proposed for the selection of control actions makes sense resulting in the chattering effect being almost suppressed even with uncertainty in the system.
\end{abstract}

Keywords. Differential inclusions, robust control, maximal monotone maps, sliding-mode control, discrete-time systems, linear uncertain systems, Lyapunov stability.

\section{Introduction}

Since its appearance in the late fifties, the so-called sliding mode motion has been associated with switching control laws. The main idea arises from the behavior of the electrical relay, i.e., the input switches between a finite number of possible values depending on the region of the phase-space in which the system is evolving. This approach works well in principle, but for real-life applications some problems arise due to the intrinsic imperfections in the elements that constitute the controller, as for example: time-delays in the reaction of the components, boundaries in the operation region (finite switching frequency), etc. Among the most dangerous effects generated because of these imperfections we can find the so-called chattering effect. The consequences of the chattering effect can be catastrophic in real systems causing component degradation, poor response and in the worst case destruction of the system.

On the other hand, the closed-loop features that offer sliding mode control are very attractive: finite-time convergence, order reduction, robustness against parametric and external disturbances, simple gain tuning. For that reason many research efforts have been directed in the study of attenuation of the chattering effect. Among these studies we can find adaptive schemes with variable gains [42], high-order sliding modes [30], regularization techniques [46], and suitable discrete-time implementation $[1,2,24,25,26,45]$.

Since the work of Filippov [20] sliding-mode control systems have been associated with differential inclusions. More precisely the solutions of a dynamical system with a discontinuous right-hand side are interpreted as solutions of an associated differential inclusion. The previous work of Filippov gives 
us the existence of solutions (in the sense of Filippov) for sliding mode control systems. Surprisingly, there are only a few studies that use the set-valued setting provided by Filippov for the design of the control law that will produce the sliding phenomenon [1, 2, 24, 25, 26, 45].

The objective of this paper is twofold. First, a family of set-valued controllers - which is suitable for the design of sliding mode controllers - is introduced using the so-called maximal monotone operators. The design procedure is revisited for the continuous-time context considering parametric uncertainty and external disturbances. It is shown that the set-valued approach is consistent with the classical design methodology and powerful, allowing us to face up the multivariable problem in a natural way as well as the regularization of the set-valued map. The second aim is to show stepby-step the methodology design for the discrete-time case when the set-valued maximal monotone operators are used together with the implicit scheme proposed in $[1,2,24]$. We show how this mathematical formulation is well-posed, providing a better understanding of discrete-time sliding mode systems.

This paper extends the results in [24] considering parametric uncertainty in the plant. It also shows that any maximal monotone set-valued map — different from the commonly used signum set-valued function - can be used in order to achieve the sliding regime. Moreover, the maximal monotone operators allow us to cover in one setting several well known formulations as the componentwise control or the unit vector control [44]. The mathematical framework used in this work for explaining the sliding mode phenomenon relies on differential inclusions, where (contrary to the conservative thinking of switching), we are making emphasis in the proper selection of the control values as the main tool towards chattering suppression.

This paper is organized as follows. In Section 2 we recall some preliminaries from convex analysis together with some notation. Section 3 is devoted to the design and well-posedness, in continuoustime, of set-valued controllers using maximal monotone operators. Some results concerned the robustness, against parametric and external disturbances, of the resulting closed-loop system are presented. The discrete-time counterpart is exposed in Section 4, where the use of the implicit discretization for achieving the discrete-time sliding phase is exposed, together with some stability results and the convergence of the solutions of the discrete-time closed-loop system to a solution of the continuous-time case. Finally, Section 5 depicts the effectiveness of the family of set-valued controllers proposed in Sections 3 and 4 through the use of a numerical example.

\section{Preliminaries and notation}

Let $X$ be a $n$-dimensional linear space, dotated with the classical Euclidean inner product denoted as $\langle\cdot, \cdot\rangle$ and the corresponding norm $\|\cdot\|$. A multivalued map $\mathbf{M}: X \rightrightarrows X$ is called monotone if it satisfies $\left\langle y_{1}-y_{2}, x_{1}-x_{2}\right\rangle \geq 0$, for all $\left(x_{1}, y_{1}\right),\left(x_{2}, y_{2}\right) \in \mathrm{Graph} \mathbf{M}$ and it is called maximal monotone if its graph is not contained in the graph of any other monotone map. The resolvent with index $\mu,(\mu>0)$, associated to a maximal monotone map $\mathbf{M}$ is a single-valued Lipschitz continuous map $J_{\mathbf{M}}^{\mu}: X \rightarrow X$ given as:

$$
J_{\mathbf{M}}^{\mu}(x):=(I+\mu \mathbf{M})^{-1}(x) .
$$

Moreover, the resolvent $J_{\mathbf{M}}^{\mu}$ is non-expansive, i.e., $\left\|J_{\mathbf{M}}^{\mu}\left(x_{1}\right)-J_{\mathbf{M}}^{\mu}\left(x_{2}\right)\right\| \leq\left\|x_{1}-x_{2}\right\|$ for all $x_{i} \in X$, $i=1,2$. A detailed study of the properties of the resolvent can be found in $[4,9,38]$. Related to the resolvent of $\mathbf{M}$ is the so-called Yosida approximation of index $\mu$ of the set-valued map $\mathbf{M}$.

Definition 1. The Yosida approximation of a maximal monotone map is given by

$$
\mathcal{M}^{\mu}(x)=\frac{1}{\mu}\left(I-J_{\mathbf{M}}^{\mu}\right)(x) .
$$

Roughly speaking, the Yosida approximation of $\mathbf{M}$ is a maximal monotone and Lipschitz continuous single-valued function which approximates the graph of $\mathbf{M}$ from below. Formally we have that for all $x \in \operatorname{Dom} \mathbf{M}$,

$$
\left\|\mathcal{M}^{\mu}(x)\right\| \leq\left\|\operatorname{Proj}_{\mathbf{M}(x)}(0)\right\|
$$


and

$$
\mathcal{M}^{\mu}(x) \rightarrow \operatorname{Proj}_{\mathbf{M}(x)}(0) \text {, as } \mu \downarrow 0 .
$$

In words, the Yosida approximation of $\mathbf{M}$ converges to the element of minimum norm in the closed convex set $\mathbf{M}(x)$. See e.g., [4, 9] for a proof of the previous statement and more properties about the Yosida approximation. The next result (taken from [4, Proposition 2, p.141]) states an important topological property concerning the graph of maximal monotone operators.

Proposition 1. The graph of a set-valued maximal monotone operator $\mathbf{M}: X \rightrightarrows X$ is stronglyweakly closed in the sense that if $x_{n} \rightarrow x$ strongly in $X$ and if $y_{n} \in \mathbf{M}\left(x_{n}\right)$ converges weakly to $y$, then $y \in \mathbf{M}(x)$.

Definition 2. Let $f: X \rightarrow \mathbb{R} \cup\{+\infty\}$ be a proper, convex, lower semicontinuous function. The subdifferential of $f$ at $x \in \operatorname{Dom} f$ is given by the set:

$$
\partial f(x):=\left\{\zeta \in X^{*} \mid\langle\zeta, \eta-x\rangle \leq f(\eta)-f(x), \text { for all } \eta \in X\right\},
$$

where $X^{*}$ refers to the dual space of $X$.

The proof of the following result can be found in [37].

Proposition 2. The subdifferential of a proper, convex, lower semicontinuous function is a maximal monotone operator.

Definition 3. Let $f: X \rightarrow \mathbb{R} \cup\{+\infty\}$ be a proper, convex, lower semicontinuous function. The proximal map $\operatorname{Prox}_{f}: X \rightarrow X$ is the unique minimizer of $f(w)+\frac{1}{2}\|x-w\|^{2}$, that is:

$$
f\left(\operatorname{Prox}_{f}(x)\right)+\frac{1}{2}\left\|x-\operatorname{Prox}_{f}(x)\right\|^{2}=\min _{w \in X}\left\{f(w)+\frac{1}{2}\|x-w\|^{2}\right\} .
$$

Along all this work we denote the identity matrix in $\mathbb{R}^{n \times n}$ as $I_{n}$. The set $\mathbb{B}_{n}:=\left\{x \in \mathbb{R}^{n} \mid\|x\| \leq 1\right\}$ represents the unit closed ball with center at the origin in $\mathbb{R}^{n}$ with the Euclidean norm. The boundary of a set $S$ is denoted $\operatorname{bd}(S)$. Let $A \in \mathbb{R}^{n \times m}$, the induced norm of the matrix $A$ is given by, $\|A\|_{m}:=\sup _{\|x\|=1}\|A x\|=\sqrt{\lambda_{\max }\left(A^{\top} A\right)}$, where $\lambda_{\max }(B):=\max _{i \in\{1, \ldots n\}}\left\{\lambda_{i} \in \sigma(B)\right\}$ and $\sigma(B)$ is the spectrum of the matrix $B \in \mathbb{R}^{n \times n}$. Let $B \in \mathbb{R}^{n \times n}$ be a symmetric matrix, $B$ is called positive definite, $(B>0)$, if for any $x \in \mathbb{R}^{n} \backslash\{0\}, x^{\top} B x>0$. It is positive semidefinite, $(B \geq 0)$, if $x^{\top} B x \geq 0$. Let $A=A^{\top}$ and $B=B^{\top}$ be square matrices, the inequality $A>B$ stands for $A-B>0$, i.e., $A-B$ is positive definite. Let $A=A^{\top}>0$, the $A$-norm of a vector $x \in \mathbb{R}^{n}$ is given by $\|x\|_{A}^{2}=x^{\top} A x$. In the case where $1 \leq p \leq \infty$ the norm $\|x\|_{p}=\left(\sum_{i}\left|x_{i}\right|^{p}\right)^{1 / p}$ for $p \in[1, \infty)$ and $\|x\|_{\infty}:=\max _{i}\left|x_{i}\right|$.

Proposition 3 (Schur's complement formula). Let $U=U^{\top} \in \mathbb{R}^{n_{1} \times n_{1}}, W=W^{\top} \in \mathbb{R}^{n_{2} \times n_{2}}$, and $R \in \mathbb{R}^{n_{1} \times n_{2}}$ be given matrices. Then, the next three statements are equivalent,

1. $\left[\begin{array}{cc}U & R \\ R^{\top} & W\end{array}\right]>0$.

2. $U>0$, and $W-R^{\top} U^{-1} R>0$.

3. $W>0$, and $U-R W^{-1} R^{\top}>0$.

\section{Design of sliding mode controllers in continuous-time using maximal monotone maps}

\subsection{The robust control problem}

In this section we make a review of the conventional methodology design of sliding mode controllers. This review will be useful for two reasons. First, we show that the family of set-valued maximal monotone operators can be used in the design of controllers that guarantee the sliding motion. 
Second, the concepts recalled here are used for introducing their discrete-time counterpart. We start analyzing a linear time-invariant system with both parametric uncertainty and external disturbances. Specifically, in this work we focus on the case in which the input matrix $B \in \mathbb{R}^{n \times m}$ is known and the dynamics of the plant is affected by a time and state-dependent additive uncertainty $\Delta_{A}(t, x) \in$ $\mathbb{R}^{n \times n}$. The system is characterized in state-space form as:

$$
\dot{x}(t)=\left(A+\Delta_{A}(t, x(t))\right) x(t)+B(u(t)+w(t, x(t))), x(0)=x_{0},
$$

where $x(t) \in \mathbb{R}^{n}$ represents the state variable, $u(t) \in \mathbb{R}^{m}$ is the control input, $w(t, x(t)) \in \mathbb{R}^{m}$ accounts for an external disturbance considered unknown but bounded in the $L^{\infty}$ sense. The matrix $A$ represents the nominal values of the parameters of the plant which are assumed to be known. Notice that, in general, the addition of the term $\Delta_{A}$ generates a state-dependent mismatched disturbance. Along all this paper, we consider the following assumptions:

Assumption 1. The pair $(A, B)$ is stabilizable.

Assumption 2. The matrix $B \in \mathbb{R}^{n \times m}$ has full column rank.

Assumption 3. For all $t \in[0,+\infty)$ the uncertainty matrix-funcion $\Delta_{A}(t, \cdot)$ is locally Lipschitz continuous and satisfies: $\Delta_{A}(t, x) \Lambda \Delta_{A}^{\top}(t, x)<I_{n}$, for all $x \in \mathbb{R}^{n}$ and for some known symmetric positive definite matrix $\Lambda \in \mathbb{R}^{n \times n}$.

Assumption 4. There exists $W>0$ such that $\sup _{t \geq 0}\|w(t, x)\| \leq W<+\infty$.

Notice that Assumption 3 implies that $\Delta_{A}(t, x)$ is uniformly bounded, since $\left\|\Delta_{A}(t, x)\right\|_{m}^{2} \leq 1 / \lambda_{\min }(\Lambda)=$ $\lambda_{\max }\left(\Lambda^{-1}\right)$ for all $(t, x) \in \mathbb{R}_{+} \times \mathbb{R}^{n}$. It is also noteworthy that the kind of parametric disturbances considered in this work embraces time-variant and a subfamily of nonlinear systems.

Proposition 4. Assumption 1 holds if and only if for some a $>0$ there exists a symmetric positive definite matrix $P \in \mathbb{R}^{n \times n}$ satisfying the following linear matrix inequality (LMI):

$$
B_{\perp}^{\top}\left(A P+P A^{\top}+2 a P\right) B_{\perp}<0,
$$

where $B_{\perp} \in \mathbb{R}^{n \times n-m}$ denotes an orthogonal complement of the matrix $B$, i.e., $B_{\perp}$ is a full column rank matrix whose columns are formed by basis vectors of the null space of $B^{\top}$.

Proof. This fact follows directly from the equation (5.17) and the elimination of matrix variables procedure described in Section 2.6.2, both in [8].

The design of sliding mode controllers is made by the selection of two main elements, the sliding surface and the control law. The former refers to a submanifold on the state-space in which all the trajectories will converge in finite-time by the action of the control law, and the closed-loop system constrained to the sliding surface satisfies the performance requirements. Moreover, once the sliding surface has been reached, the task of the controller is to maintain the trajectories inside it despite the presence of disturbances (sliding phase). In this work the design of the control law is performed using a two-step design methodology. Namely, in the former stage we compute a nominal control, denoted as $u^{\text {nom }}$, that guarantees the invariance of the sliding surface $\sigma=0$ in the absence of the uncertainties, i.e., $w \equiv 0$ and $\Delta_{A} \equiv 0_{n \times n}$. After that, we propose the set-valued component of the controller, denoted by $u^{\mathrm{sv}}$, which will be responsible for attaining the sliding surface as well as providing robustness against matched disturbances. A crucial point to consider is related to the proper design of the sliding surface which will guarantee the performance of the system in the sliding phase. It was proved in $[14,16,36]$ that the correct design of the sliding surface helps to diminish the effects caused by mismatched disturbances and in some special cases (when some structure of the disturbance is imposed) even suppression can be accomplished [17]. More important is the fact that the wrong selection of this surface could increase the effects of the disturbance [14], which in our context implies higher gains. Throughout this work we consider the sliding surface as a hyperplane of the form $\sigma=C x$.

Assumption 5. The matrix $C \in \mathbb{R}^{m \times n}$ is such that the product $C B$ is nonsingular. 
Assumption 5 will guarantee the uniqueness of the equivalent control as well as the uniqueness of the nominal control. It is noteworthy that the two-step design methodology described above is sometimes called equivalent-control-based method and the part of the controller denoted by $u^{\text {nom }}$ is called the equivalent control. In this work the concept of equivalent control is used as in [44], i.e., it is the control that maintains the state in sliding motion in the presence of disturbances. It follows that the term $u^{\text {nom }}$ is a nominal equivalent control, but we prefer called it merely nominal in order to avoid confusion.

\subsection{Design of the sliding surface}

In this subsection we follow the lines of [14], analyzing the effect of the sliding surface $\sigma=C x$ over the mismatched disturbance. We start studying how the dynamics in sliding phase is affected by the disturbance $\Delta_{A}(t, x) x$. To this end we use the equivalent control method [41]. Namely, we compute the control that maintains the sliding regime and we will see how the mismatched disturbance affects the closed-loop system. The equivalent control is computed from the invariance condition $\dot{\sigma}=0$ as:

$$
\left.C\left(A x+B\left(u^{\mathrm{eq}}+w\right)\right)+\Delta_{A}\left(t, x^{\mathrm{eq}}\right) x^{\mathrm{eq}}\right)=0, \Rightarrow u^{\mathrm{eq}}=-(C B)^{-1} C\left(A x^{\mathrm{eq}}+\Delta_{A}\left(t, x^{\mathrm{eq}}\right) x^{\mathrm{eq}}\right)-w .
$$

Substitution of the equivalent control into (4) leads to the expression of the dynamics in sliding phase,

$$
\dot{x}^{\mathrm{eq}}=\left(I_{n}-B(C B)^{-1} C\right) A x^{\mathrm{eq}}+\left(I_{n}-B(C B)^{-1} C\right) \Delta_{A}\left(t, x^{\mathrm{eq}}\right) x^{\mathrm{eq}},
$$

from which it becomes clear that the matrix characterizing the sliding hyperplane plays a role into the equivalent disturbance $\left(I_{n}-B(C B)^{-1} C\right) \Delta_{A}(t, x) x$. In [14] the authors proved that the correct design of such hyperplane guarantees the no amplification of the disturbance by using surfaces of the form $C=B^{\top}$ or $C=B^{+}$, where $B^{+}$stands for the left-inverse of the matrix $B$, i.e., $B^{+}=\left(B^{\top} B\right)^{-1} B^{-1}$. In this work we modify such selection of the surface considering instead $C=B^{\top} P^{-1}$ and also $C=\left(B^{\top} P^{-1} B\right)^{-1} B^{\top} P^{-1}$, where $P$ is a solution of (5). First we show that this selection of $C$ gives us an equivalent disturbance with minimum $P^{-1}$-norm. Afterwards we show how the proper choice of the matrix $P$ dominates the mismatched disturbance in sliding phase.

Lemma 1. Let $C_{1}=B^{\top} P^{-1}$ and $C_{2}=\left(B^{\top} P^{-1} B\right)^{-1} B^{\top} P^{-1}$, then both $C_{i}, i=1,2$, minimize the $P^{-1}$-norm of the equivalent disturbance $\left(I_{n}-B(C-B)^{-1} C\right) \Delta_{A}\left(t, x^{\mathrm{eq}}\right) x^{\mathrm{eq}}$.

Proof. Let $\phi^{\mathrm{eq}}=\Delta A\left(t, x^{\mathrm{eq}}\right) x^{\mathrm{eq}}$. Then, the optimization problem

$$
\min _{C \in \mathbb{R}^{m \times n}}\left\|\left(I_{n}-B(C B)^{-1} C\right) \phi^{\mathrm{eq}}\right\|_{P^{-1}}^{2}=\min _{z \in \mathbb{R}^{m}}\left\|\phi^{\mathrm{eq}}-B z\right\|_{P^{-1}}^{2},
$$

where $z=(C B)^{-1} C \phi^{\text {eq }}$, has a unique solution given by $z^{*}=\left(B^{\top} P^{-1} B\right)^{-1} B^{\top} P^{-1} \phi^{\text {eq }}$. From the definition of $z$ it follows that $C=B^{\top} P^{-1}$ achieves the minimum in (8), as well as, $C=$ $\left(B^{\top} P^{-1} B\right)^{-1} B^{\top} P^{-1}$.

Notice that both selections of $C$ stated in Lemma 1 satisfy Assumption 5. Throughout this section we will set $C=\left(B^{\top} P^{-1} B\right)^{-1} B^{\top} P^{-1}$. In the next subsection we design the control law that assures the sliding motion.

\subsection{Design of the control law}

The computation of the nominal control is made from the invariance condition $\dot{\sigma}=0$ in the ideal case, (i.e., $w=0, u^{\mathrm{sv}}=0$ and $\Delta_{A}=0$ ), as:

$$
\dot{\sigma}=C \dot{x}=C\left(A x+B u^{\text {nom }}\right)=0 \Rightarrow u^{\text {nom }}=-(C B)^{-1} C A x .
$$

Notice that the nominal control is nothing more than a linear feedback law of the form $u^{\text {nom }}=-\Gamma x$ with $\Gamma=(C B)^{-1} C A$. Substitution of the nominal control (9) into the system (4) yields,

$$
\dot{x}=\left(I_{n}-B(C B)^{-1} C\right) A x+B\left(u^{\mathrm{sv}}+w\right)+\Delta_{A}(t, x) x,
$$


where $u^{\mathrm{sv}}$ is the set-valued part of the controller. In order to obtain the dynamics of the system in the sliding phase, we consider the nonsingular transformation,

$$
T=\left[\begin{array}{c}
B_{\perp}^{\top} \\
\left(B^{\top} P^{-1} B\right)^{-1} B^{\top} P^{-1}
\end{array}\right], T^{-1}=\left[P B_{\perp}\left(B_{\perp}^{\top} P B_{\perp}\right)^{-1} \quad B\right] .
$$

Remark 1. It is worth to mention that from the product $T^{-1} T$ we obtain the identity,

$$
P B_{\perp}\left(B_{\perp}^{\top} P B_{\perp}\right)^{-1} B_{\perp}^{\top}+B\left(B^{\top} P^{-1} B\right)^{-1} B^{\top} P^{-1}=I_{n} .
$$

From the application of (12) to the term $\phi:=\Delta_{A}(t, x) x$ it follows that

$$
\phi=P B_{\perp}\left(B_{\perp}^{\top} P B_{\perp}\right)^{-1} B_{\perp}^{\top} \phi+B\left(B^{\top} P^{-1} B\right)^{-1} B^{\top} P^{-1} \phi=P B_{\perp} \phi_{u}+B \phi_{m},
$$

where, $\phi_{u}:=\left(B_{\perp}^{\top} P B_{\perp}\right)^{-1} B_{\perp}^{\top} \phi$ and $\phi_{m}:=\left(B^{\top} P^{-1} B\right)^{-1} B^{\top} P^{-1} \phi$ are called the unmatched and the matched parts of $\phi$ respectively.

The change of coordinates $z=T x$ leads to the regular form [44],

$$
\left\{\begin{array}{l}
\dot{z}_{1}=B_{\perp}^{\top}\left(A+\hat{\Delta}_{A}(t, z)\right) P B_{\perp}\left(B_{\perp}^{\top} P B_{\perp}\right)^{-1} z_{1}+B_{\perp}^{\top}\left(A+\hat{\Delta}_{A}(t, z)\right) B \sigma \\
\dot{\sigma}=u^{\mathrm{sv}}+\hat{w}(t, z)+\hat{\phi}_{m}(t, z),
\end{array}\right.
$$

where, $\hat{\Delta}_{A}(t, z):=\Delta_{A}\left(t, T^{-1} z\right), \hat{w}(t, z):=w\left(t, T^{-1} z\right)$ and $\hat{\phi}_{m}(t, z):=\phi_{m}\left(t, T^{-1} z\right)$. One comment takes place here. First, it is easy to see that $z_{2}=\sigma$ and from (13b) it follows that the dynamics of the sliding variable is only affected by the matched part of the original disturbance $\Delta_{A}(t, x) x$. Hence, in order to achieve the sliding regime it is necessary to take into account only the matched part of the disturbance in the design of $u^{\mathrm{sv}}$ [14].

In the next lines we show what are the conditions that the matrix $P$ must satisfy such that the reduced order dynamics $z_{1}$ is asymptotically stable with decay rate $a$, in the ideal sliding phase, under the influence of the parametric uncertainty $\Delta_{A}$. To this end, let us consider the reduced order system

$$
\dot{z}_{1}=B_{\perp}^{\top}\left(A+\hat{\Delta}_{A}(t, z)\right) P B_{\perp}\left(B_{\perp}^{\top} P B_{\perp}\right)^{-1} z_{1},
$$

with the Lyapunov-function candidate $V\left(z_{1}\right)=\frac{1}{2} z_{1}^{\top}\left(B_{\perp}^{\top} P B_{\perp}\right)^{-1} z_{1}$. Taking the derivative of $V$ along the trajectories of (14) yields,

$$
\begin{aligned}
\dot{V} & =z_{1}^{\top}\left(B_{\perp}^{\top} P B_{\perp}\right)^{-1} \dot{z}_{1} \\
& =\frac{1}{2} \bar{z}_{1}^{\top} B_{\perp}^{\top}\left(A P+P A^{\top}\right) B_{\perp} \bar{z}_{1}+\bar{z}_{1}^{\top} B_{\perp}^{\top} \Delta_{A} P B_{\perp} \bar{z}_{1},
\end{aligned}
$$

where $\bar{z}_{1}=\left(B_{\perp}^{\top} P B_{\perp}\right)^{-1} z_{1}$. Applying (5), together with the inequality $2 p^{\top} X^{\top} Y q \leq p^{\top} X^{\top} \Psi X p+$ $q^{\top} Y^{\top} \Psi^{-1} Y q$, for some $\Psi=\Psi^{\top}>0$, it follows that

$$
\dot{V} \leq-a \bar{z}_{1}^{\top} P \bar{z}_{1}+\frac{1}{2} \bar{z}_{1}^{\top} B_{\perp}^{\top} \Delta_{A} \Psi \Delta_{A}^{\top} B_{\perp} \bar{z}_{1}+\frac{1}{2} \bar{z}_{1} B_{\perp}^{\top} P \Psi^{-1} P B_{\perp} \bar{z}_{1} .
$$

Taking $\Psi=\Lambda$ where $\Lambda=\Lambda^{\top}>0$ is defined in Assumption 3 gives,

$$
\begin{aligned}
\dot{V} & \leq-a \bar{z}_{1}^{\top} P \bar{z}_{1}+\frac{1}{2} \bar{z}_{1}^{\top} B_{\perp}^{\top} B_{\perp} \bar{z}_{1}+\frac{1}{2} \bar{z}_{1} B_{\perp}^{\top} P \Lambda^{-1} P B_{\perp} \bar{z}_{1} \\
& =-\bar{z}_{1}^{\top} B_{\perp}^{\top}\left(a P-\frac{1}{2} I_{n}-\frac{1}{2} P \Lambda^{-1} P\right) B_{\perp} \bar{z}_{1} .
\end{aligned}
$$

From (17) asymptotic stability of the reduced system (14) in sliding phase follows if

$$
B_{\perp}^{\top}\left(a P-\frac{1}{2} I_{n}-\frac{1}{2} P \Lambda^{-1} P\right) B_{\perp}>0,
$$


Along all this section we will assume that the matrix $P$ satisfies (5) and a stronger version of (18). Namely,

$$
Q:=\left[\begin{array}{cc}
B_{\perp}^{\top}\left(a P-I_{n}-\frac{1}{2} P \Lambda^{-1} P\right) B_{\perp} & -\frac{1}{2} B_{\perp}^{\top} A B \\
-\frac{1}{2} B^{\top} A^{\top} B_{\perp} & K-\frac{1}{2} B^{\top} \Lambda^{-1} B
\end{array}\right]>0,
$$

where $K=K^{\top} \in \mathbb{R}^{m \times m}$ is a positive definite matrix introduced below. Applying Schur's complement formula, it is easy to show that (19) is equivalent to

$$
\left[\begin{array}{cccc}
B_{\perp}^{\top}\left(a P-I_{n}\right) B_{\perp} & -\frac{1}{2} B_{\perp}^{\top} A B & B_{\perp}^{\top} P & 0_{n-m \times n} \\
-\frac{1}{2} B^{\top} A^{\top} B_{\perp} & K & 0_{m \times n} & B^{\top} \\
P B_{\perp} & 0_{n \times m} & 2 \Lambda & 0_{n \times n} \\
0_{n \times n-m} & B & 0_{n \times n} & 2 \Lambda
\end{array}\right]>0 .
$$

The justification for considering (19) instead of (18) comes from the proof of the Theorem 1 where the complete system (13) is analyzed. Remark that the LMI (20) is feasible for $a>0$ big enough and $K, \Lambda$ sufficiently big too. This last condition translates to considering small parametric uncertainties $\Delta_{A}$, see Assumption 3.

Proposition 5. The disturbance term $\hat{\phi}_{m}(t, z)$ satisfies the linear growth condition $\left\|\hat{\phi}_{m}(t, z)\right\| \leq$ $\sqrt{\kappa}\|z\|$, where

$$
\kappa=\frac{\lambda_{\max }(P) \lambda_{\max }\left(\Lambda^{-1}\right)}{\lambda_{\min }\left(B^{\top} P^{-1} B\right) \lambda_{\min }(P)} \max \left\{\frac{1}{\lambda_{\min }\left(B_{\perp}^{\top} P B_{\perp}\right)}, \lambda_{\max }\left(B^{\top} P^{-1} B\right)\right\}
$$

Proof. From the definition of $\hat{\phi}_{m}$ we have that

$$
\begin{aligned}
\left\|\hat{\phi}_{m}(t, z)\right\| & =\left\|\left(B^{\top} P^{-1} B\right)^{-1} B^{\top} P^{-1} \hat{\Delta}_{A}(t, z) T^{-1} z\right\| \\
& \leq\left\|\left(B^{\top} P^{-1} B\right)^{-1} B^{\top} P^{-1 / 2}\right\|_{m}\left\|P^{-1 / 2}\right\|_{m}\left\|\hat{\Delta}_{A}(t, z)\right\|_{m}\left\|T^{-1}\right\|_{m}\|z\| .
\end{aligned}
$$

Recalling that the induced euclidean norm coincides with the spectral norm and making use of the Assumption 3, after simple computations we obtain,

$$
\left\|\hat{\phi}_{m}(t, z)\right\| \leq \sqrt{\frac{\lambda_{\max }\left(\Lambda^{-1}\right)}{\lambda_{\min }\left(B^{\top} P^{-1} B\right) \lambda_{\min }(P)}}\left\|T^{-1}\right\|_{m}\|z\| .
$$

On the other hand, from (11) it follows that,

$$
\begin{aligned}
\left\|T^{-\top}\right\|_{m}^{2} & \leq\left\|\left[\begin{array}{c}
\left(B_{\perp}^{\top} P B_{\perp}\right)^{-1} B_{\perp}^{\top} P^{1 / 2} \\
B^{\top} P^{-1 / 2}
\end{array}\right]\right\|_{m}^{2}\left\|P^{1 / 2}\right\|_{m}^{2} \\
& =\lambda_{\max }(P) \lambda_{\max }\left(\left[\begin{array}{cc}
\left(B_{\perp}^{\top} P B_{\perp}\right)^{-1} & 0 \\
0 & B^{\top} P^{-1} B
\end{array}\right]\right),
\end{aligned}
$$

and the result follows. This concludes the proof.

\subsubsection{Set-valued controller}

In this subsection we study the family of set-valued maximal monotone operators used as feedback control laws for the system (13). First, some results about the existence and (in some cases) uniqueness of solutions are presented. Subsequently, we prove how a subfamily of the family of maximal monotone controllers yields finite-time stable sliding modes. We start setting the missing term $u^{\mathrm{sv}}$ in $(13 \mathrm{~b})$ as,

$$
-u^{\mathrm{sv}}(t) \in K \sigma(t)+\gamma(z(t)) \mathbf{M}(\sigma(t))
$$

where $K \in \mathbb{R}^{m \times m}$ is a positive definite matrix satisfying (20), $\gamma: \mathbb{R}^{n} \rightarrow \mathbb{R}_{+}$is a positive function depending on the system state $z$, and $\mathbf{M}: \mathbb{R}^{m} \rightrightarrows \mathbb{R}^{m}$ is a set-valued maximal monotone operator. 
Thus, from (22) it follows that there exists $\zeta \in \mathbf{M}(\sigma)$ such that $-u^{\mathrm{sv}}=K z+\gamma(z) \zeta$. Hence, the evolution of the sliding variable is dictated by the following differential inclusion,

$$
\left\{\begin{array}{l}
\dot{\sigma}(t)=-K \sigma(t)-\gamma(z(t)) \zeta(t)+\hat{w}(t, z)+\hat{\phi}_{m}(t, z), \sigma(0)=\sigma_{0} \\
\zeta(t) \in \mathbf{M}(\sigma(t)) .
\end{array}\right.
$$

In the case when the function $\gamma$ is constant, the differential inclusion (23) belongs to the class of differential inclusions with maximal monotone right-hand side for which numerous results have been proposed, see e.g., [4, 6, 9, 10, 12, 33, 35] and it embraces several mathematical formulations [11]. The existence and uniqueness of solutions of (23) for the case where $\gamma$ is constant has been studied for several conditions imposed on the term $\hat{w}+\hat{\phi}$, see e.g., $[9,12,15]$. For a solution of (23) we mean an absolutely continuous function $\sigma: \mathbb{R}_{+} \rightarrow \mathbb{R}^{m}$ that satisfies $\sigma(0)=\sigma_{0} \in$ Dom $\mathbf{M}$ together with (23) almost everywhere on $[0,+\infty)$, that is, we consider solutions of differential inclusion (23) in the sense of Caratheodory [18]. It is worth to mention that in the case where $\gamma$ is a function of the state, the uniqueness of solutions of (23) is not guaranteed, this comes from the fact that, in general, the map $\gamma(z) M(\sigma)$ is not maximal monotone. Here, we present some examples about the different choices of the set-valued map $\mathbf{M}$.

Example 1. Let $\mathbf{M}$ be the subdifferential of $f(\sigma):=\|\sigma\|_{1}=\sum_{i=1}^{n}\left|\sigma_{i}\right|$. Then, $\mathbf{M}(\sigma)$, is the vector set-valued signum function,

$$
[\mathbf{M}(\sigma)]_{i}= \begin{cases}1, & \text { if } \sigma_{i}>0 \\ {[-1,1],} & \text { if } \sigma_{i}=0, \\ -1 & \text { if } \sigma_{i}<0 .\end{cases}
$$

In this case the control scheme agrees with the so-called componentwise sliding mode design, see e.g., [44].

Example 2. Let $\mathbf{M}$ be the subdifferential of $f(\sigma):=\|\sigma\|_{2}$. Then $\mathbf{M}(\sigma)$ is the set-valued vector function,

$$
\mathbf{M}(\sigma)=\left\{\begin{array}{cl}
\mathbb{B}_{n}, & \text { if }\|\sigma\|=0, \\
\frac{\sigma}{\|\sigma\|}, & \text { otherwise. }
\end{array}\right.
$$

In this case the control scheme coincides with the so-called unit vector approach [34, 39].

Example 3. Let $\Psi_{S}$ be the indicator function of the closed convex set $S$, i.e., $\Psi_{S}(\sigma)=0$, if $\sigma \in S$ and $\Psi_{S}(\sigma)=+\infty$ otherwise. Let $\sigma(0)$ be inside the set $S$ and let $\mathbf{M}$ be the subdifferential of the indicator function,

$$
\mathbf{M}(\sigma)=\left\{\zeta \in \mathbb{R}^{m} \mid\langle\zeta, \eta-\sigma\rangle \leq 0, \text { for all } \eta \in S\right\}=N_{S}(\sigma) .
$$

Here $N_{S}(\sigma)$ denotes the normal cone to the set $S$ at the point $\sigma$. Then the closed-loop system (13b)(22) is well-posed and by Theorem 2 below the sliding mode is reached in finite time. The study of this kind of controllers has been reported in [31, 32]. Moreover, if $S=S(t)$ is a Lipschitz continuous set-valued mapping, then the closed-loop system (13b)-(22) represents a perturbed Moreau's sweeping process [13, 19].

In what follows we consider the next condition on the set-valued operator $\mathbf{M}$.

Assumption 6. The set-valued maximal monotone map $\mathbf{M}$ satisfies: $0 \in \operatorname{int} \mathbf{M}(0)$.

Remark 2. Assumption 6 is known as a condition for dry friction in the mechanics literature. It is strongly linked to the finite-time convergence property, see Theorem 2 and Corollary 3 below. In $[3,5]$ the same condition was used for proving the finite-time stability of nonlinear oscillators in both, continuous and discrete-time settings.

It is worth to mention that Assumption 6 rules out linear controllers, since we ask for maps $\mathbf{M}$ that must be set-valued at the origin. For example, in the case when $\mathbf{M}=\partial \Phi$ where the function $\Phi$ is 
proper, convex and lower semicontinuous, Assumption 6 ask for functions $\Phi$ which are nonsmooth at the origin, so that int $\mathbf{M}(0) \neq \emptyset$, as for example, the norm function $\|\cdot\|_{p}, 1 \leq p \leq \infty$. This last comment reveals that the maximal monotone operators suit perfectly as a tool that unifies the different generalizations of the signum multifunction in the design of sliding mode controllers in the multivariable case.

Proposition 6. Let Assumption 6 hold. Then for any $(x, y) \in \mathrm{Graph} \mathbf{M}$ there exists an $\varepsilon>0$ such that,

$$
\langle x, y\rangle \geq \varepsilon\|x\| .
$$

Proof. From Assumption 6, it follows that there exists $\varepsilon>0$ such that for all $\rho \in \varepsilon \mathbb{B}_{m}(0, \rho) \in$ Graph M. Then, from the definition of a maximal monotone map it follows that for any $(x, y) \in$ Graph $\mathbf{M}$ and any $\rho \in \varepsilon \mathbb{B}_{m}, 0 \leq\langle y-\rho, x\rangle$. Consequently, $\sup _{\rho \in \varepsilon \mathbb{B}_{m}}\langle\rho, x\rangle \leq\langle y, x\rangle$. The conclusion follows.

\subsection{Well-posedness and stability of the closed-loop system}

In this subsection we show the well-posedness of the closed-loop system $(13),(22)$ in the case when $\gamma$ is a state-dependent gain by imposing some conditions on $P$, in the form of LMI's, such that the unmatched part of the disturbance is dominated, and hence assuring the asymptotic stability of the fixed-point $z_{1}^{*}=0$. After that, we show how the sliding phase is reached in finite time with an appropriate selection of the gain $\gamma$. Finally some results about stability and uniqueness of solutions in the case where $\gamma$ is constant, are established.

Theorem 1. Let Assumptions 1-6 hold. Then the closed-loop system (13),(22), where $\mathbf{M}:$ Dom $\mathbf{M} \rightrightarrows$ $\mathbb{R}^{m}$ is a set-valued maximal monotone map that satisfies Dom $\mathbf{M}=\mathbb{R}^{m}$, has at least one solution (in Caratheodory's sense [18]), whenever, $P=P^{\top}>0$ satisfies the LMI's (5), (20) and in addition for some $\rho>0$,

$$
\varepsilon \gamma(z)=\rho+W+\sqrt{\kappa}\|z(t)\|,
$$

where $\kappa$ is as in (21) and $\varepsilon>0$ is such that $\varepsilon \mathbb{B}_{m} \subset \mathbf{M}(0)$.

Proof. The proof follows a classical approach. Namely, first we approximate the solutions of the differential inclusion (13),(22) by using differential equations. After that, the boundedness of the solutions of the differential equation for all time $t \in[0,+\infty)$ is proved. Finally, the application of the Arzelà-Ascoli [29, Theorem 1.3.8] and the Banach-Alaoglu [29, Theorem 2.4.3] theorems gives us the convergence of the sequence formed from the solutions of the differential equation to one solution of the differential inclusion (13),(22), see e.g., [3]. We start with the proof as follows, consider first the differential equation

$$
\left\{\begin{array}{l}
\dot{z}_{1}^{\mu}=B_{\perp}^{\top}\left(A+\hat{\Delta}_{A}\left(t, z^{\mu}\right)\right) P B_{\perp}\left(B_{\perp}^{\top} P B_{\perp}\right)^{-1} z_{1}^{\mu}+B_{\perp}^{\top}\left(A+\hat{\Delta}_{A}\left(t, z^{\mu}\right)\right) B \sigma^{\mu} \\
\dot{\sigma}^{\mu}=-K \sigma^{\mu}+\hat{w}\left(t, z^{\mu}\right)+\hat{\phi}_{m}\left(t, z^{\mu}\right)-\gamma\left(z^{\mu}\right) \mathcal{M}^{\mu}\left(\sigma^{\mu}\right)
\end{array}\right.
$$

where $z^{\mu}=\left[z_{1}^{\mu \top} \sigma^{\mu \top}\right]^{\top}$ and the map $\mathcal{M}^{\mu}: \mathbb{R}^{m} \rightarrow \mathbb{R}^{m}$ refers to the Yosida approximation of index $\mu>0$ of the map $\mathbf{M}$ (see Definition 1). It is a well known fact that the Yosida approximation is a Lipschitz continuous function with constant $1 / \mu$. Hence, it follows that there exists one solution to (26) in $[0, T)$ for some $T>0$. Next, using a Lyapunov analysis we show that the solution of (26) exists for all time $t>0$. To this end, consider the positive definite function

$$
V\left(z_{1}^{\mu}, \sigma^{\mu}\right):=\frac{1}{2} z_{1}^{\mu \top}\left(B_{\perp}^{\top} P B_{\perp}\right)^{-1} z_{1}^{\mu}+\frac{1}{2} \sigma^{\mu \top} \sigma^{\mu},
$$

where we recall that $B_{\perp}$ is full column rank and hence $B_{\perp}^{\top} P B_{\perp}>0$. Deriving $V$ along the trajectories 
of (26) leads to,

$$
\begin{aligned}
\dot{V}= & z_{1}^{\mu \top}\left(B_{\perp}^{\top} P B_{\perp}\right)^{-1} \dot{z}_{1}^{\mu}+\sigma^{\mu \top} \dot{\sigma}^{\mu} \\
= & z_{1}^{\mu \top}\left(B_{\perp}^{\top} P B_{\perp}\right)^{-1} B_{\perp}^{\top}\left(A+\hat{\Delta}_{A}\left(t, z^{\mu}\right)\right) P B_{\perp}\left(B_{\perp}^{\top} P B_{\perp}\right)^{-1} z_{1}^{\mu} \\
& +z_{1}^{\mu \top}\left(B_{\perp}^{\top} P B_{\perp}\right)^{-1} B_{\perp}^{\top}\left(A+\hat{\Delta}_{A}\left(t, z^{\mu}\right)\right) B \sigma^{\mu}-\sigma^{\mu \top} K \sigma^{\mu} \\
& +\sigma^{\mu \top}\left(-\gamma\left(z^{\mu}\right) \mathcal{M}^{\mu}\left(\sigma^{\mu}\right)+\hat{w}\left(t, z^{\mu}\right)+\hat{\phi}_{m}\left(t, z^{\mu}\right)\right) \\
\leq & \frac{1}{2} \bar{z}_{1}^{\mu \top} B_{\perp}^{\top}\left(A P+P A^{\top}\right) B_{\perp} \bar{z}_{1}^{\mu}+\bar{z}_{1}^{\mu \top} B_{\perp}^{\top} A B \sigma^{\mu}+\bar{z}_{1}^{\mu \top} B_{\perp}^{\top} \hat{\Delta}_{A}\left(t, z^{\mu}\right) P B_{\perp} \bar{z}_{1}^{\mu} \\
& +\bar{z}_{1}^{\mu \top} B_{\perp}^{\top} \hat{\Delta}_{A}\left(t, z^{\mu}\right) B \sigma^{\mu}-\sigma^{\mu \top} K \sigma^{\mu}+\sigma^{\mu \top}\left(-\gamma\left(z^{\mu}\right) \mathcal{M}^{\mu}\left(\sigma^{\mu}\right)+\hat{w}\left(t, z^{\mu}\right)+\hat{\phi}_{m}\left(t, z^{\mu}\right)\right),
\end{aligned}
$$

where, $\bar{z}_{1}^{\mu}=\left(B_{\perp}^{\top} P B_{\perp}\right)^{-1} z_{1}^{\mu}$. The next step consists in finding bounds for the terms that involve the unknown matrix $\hat{\Delta}_{A}$. Using the inequality $2 p^{\top} X^{\top} Y q \leq p^{\top} X^{\top} \Psi X p+q^{\top} Y^{\top} \Psi^{-1} Y q$, where $\Psi=\Psi^{\top}>0$, gives us the bounds

$$
\begin{aligned}
\bar{z}_{1}^{\mu \top} B_{\perp}^{\top} \hat{\Delta}_{A} P B_{\perp} \bar{z}_{1}^{\mu} & \leq \frac{1}{2} \bar{z}_{1}^{\mu \top} B_{\perp}^{\top} \hat{\Delta}_{A} \Psi \hat{\Delta}_{A}^{\top} B_{\perp} \bar{z}_{1}^{\mu}+\frac{1}{2} \bar{z}_{1}^{\mu \top} B_{\perp}^{\top} P \Psi^{-1} P B_{\perp} \bar{z}_{1}^{\mu} \\
\bar{z}_{1}^{\mu \top} B_{\perp}^{\top} \hat{\Delta}_{A} B \sigma^{\mu} & \leq \frac{1}{2} \bar{z}_{1}^{\mu \top} B_{\perp}^{\top} \hat{\Delta}_{A} \Psi \hat{\Delta}_{A}^{\top} B_{\perp} \bar{z}_{1}^{\mu}+\frac{1}{2} \sigma^{\mu \top} B^{\top} \Psi^{-1} B \sigma^{\mu} .
\end{aligned}
$$

Taking $\Psi=\Lambda$ where $\Lambda=\Lambda^{\top}>0$ satisfies Assumption 3, the substitution of (29)-(30) into (28) yields:

$$
\begin{aligned}
\dot{V} \leq & -\bar{z}_{1}^{\mu \top} B_{\perp}^{\top}\left(a P-I_{n}-\frac{1}{2} P \Lambda^{-1} P\right) B_{\perp} \bar{z}_{1}^{\mu}+\bar{z}_{1}^{\top} B_{\perp}^{\top} A B \sigma-\sigma^{\mu \top}\left(K-\frac{1}{2} B^{\top} \Lambda^{-1} B\right) \sigma^{\mu} \\
& +\sigma^{\mu \top}\left(-\gamma\left(z^{\mu}\right) \mathcal{M}^{\mu}\left(\sigma^{\mu}\right)+\hat{w}\left(t, z^{\mu}\right)+\hat{\phi}_{m}\left(t, z^{\mu}\right)\right) \\
\leq & -\lambda_{\min }(\tilde{Q})\|z\|^{2}-\gamma\left(z^{\mu}\right) \sigma^{\mu \top} \mathcal{M}^{\mu}\left(\sigma^{\mu}\right)+\left(W+\sqrt{\kappa}\left\|z^{\mu}\right\|\right)\left\|\sigma^{\mu}\right\|
\end{aligned}
$$

where $\tilde{Q} \in \mathbb{R}^{n \times n}$ is given as

$$
\tilde{Q}:=\left[\begin{array}{cc}
\left(B_{\perp}^{\top} P B_{\perp}\right)^{-1} & 0 \\
0 & I_{m}
\end{array}\right] Q\left[\begin{array}{cc}
\left(B_{\perp}^{\top} P B_{\perp}\right)^{-1} & 0 \\
0 & I_{m}
\end{array}\right]>0
$$

and $Q$ is defined in (19). We proceed to analyze the term $\left\langle\sigma^{\mu}, \mathcal{M}^{\mu}\left(\sigma^{\mu}\right)\right\rangle$ as follows. From the definition of the Yosida approximation (Definition 1 in Section 2) we have that $\sigma^{\mu}=\mu \mathcal{M}^{\mu}\left(\sigma^{\mu}\right)+$ $J_{\mathbf{M}}^{\mu}\left(\sigma^{\mu}\right)$, and $\left(J_{\mathbf{M}}^{\mu}\left(\sigma^{\mu}\right), \mathcal{M}^{\mu}\left(\sigma^{\mu}\right)\right) \in$ Graph $\mathbf{M}$. Hence, making use of both previous facts together with (24) in Proposition 6 yields,

$$
\begin{aligned}
\left\langle\sigma^{\mu}, \mathcal{M}^{\mu}\left(\sigma^{\mu}\right)\right\rangle & =\mu\left\|\mathcal{M}\left(\sigma^{\mu}\right)\right\|^{2}+\left\langle J_{\mathbf{M}}^{\mu}\left(\sigma^{\mu}\right), \mathcal{M}^{\mu}\left(\sigma^{\mu}\right)\right\rangle \\
& \geq \mu\left\|\mathcal{M}\left(\sigma^{\mu}\right)\right\|^{2}+\varepsilon\left\|J_{\mathbf{M}}^{\mu}\left(\sigma^{\mu}\right)\right\| \\
& =\mu\left\|\mathcal{M}\left(\sigma^{\mu}\right)\right\|^{2}+\varepsilon\left\|\sigma^{\mu}-\mu \mathcal{M}^{\mu}\left(\sigma^{\mu}\right)\right\| \\
& \geq \varepsilon\left\|\sigma^{\mu}\right\|+\mu\left\|\mathcal{M}^{\mu}\left(\sigma^{\mu}\right)\right\|\left(\left\|\mathcal{M}^{\mu}\left(\sigma^{\mu}\right)\right\|-\varepsilon\right) .
\end{aligned}
$$

Substitution of (34) into (32) results in

$$
\begin{aligned}
\dot{V} & \leq-\lambda_{\min }(\tilde{Q})\left\|z^{\mu}\right\|^{2}+\left\|\sigma^{\mu}\right\|\left(W+\sqrt{\kappa}\left\|z^{\mu}\right\|\right)-\gamma\left(z^{\mu}\right)\left(\varepsilon\left\|\sigma^{\mu}\right\|+\mu\left\|\mathcal{M}^{\mu}\left(\sigma^{\mu}\right)\right\|\left(\left\|\mathcal{M}^{\mu}\left(\sigma^{\mu}\right)\right\|-\varepsilon\right)\right) \\
& \left.\leq-\lambda_{\min }(\tilde{Q})\left\|z^{\mu}\right\|^{2}-\left(\varepsilon \gamma\left(z^{\mu}\right)-W-\sqrt{\kappa}\left\|z^{\mu}\right\|\right)\left\|\sigma^{\mu}\right\|-\gamma\left(z^{\mu}\right) \mu\left\|\mathcal{M}^{\mu}\left(\sigma^{\mu}\right)\right\|\left(\left\|\mathcal{M}^{\mu}\left(\sigma^{\mu}\right)\right\|-\varepsilon\right)\right) .
\end{aligned}
$$

Now we continue with the proof showing that for all $\sigma^{\mu} \notin \mu \varepsilon \mathbb{B}_{m}$ the term $\left\|\mathcal{M}^{\mu}\left(\sigma^{\mu}\right)\right\|-\varepsilon$ is nonnegative. To this end, first notice that for any $v \in \mu \varepsilon \mathbb{B}_{m} \subset \mu \mathbf{M}(0)$, the resolvent $J_{\mathbf{M}}^{\mu}$ at the point $v$ is zero. Indeed, let $\varepsilon>0$ be such that $\varepsilon \mathbb{B}_{m} \subset \mathbf{M}(0)$. Then, it follows that for any $v \in \mu \varepsilon \mathbb{B}_{m}$, $v \in \mu \mathbf{M}(0)=(I+\mu \mathbf{M})(0)$. Therefore, $J_{\mathbf{M}}^{\mu}(v)=0$. From the non-expansiveness property of the 
resolvent it follows that $\left\|J_{\mathbf{M}}^{\mu}\left(\sigma^{\mu}\right)\right\| \leq\left\|\sigma^{\mu}-v\right\|$, for all $v \in \mu \varepsilon \mathbb{B}_{m}$. So, from the definition of the Yosida approximation, taking $v=\mu \varepsilon \frac{\sigma^{\mu}}{\left\|\sigma^{\mu}\right\|}$, and recalling that we are analyzing the case where $\left\|\sigma^{\mu}\right\| \geq \mu \varepsilon$, we have,

$$
\begin{aligned}
\left\|\mathcal{M}^{\mu}\left(\sigma^{\mu}\right)\right\| & =\frac{1}{\mu}\left\|\sigma^{\mu}-J_{\mathbf{M}}^{\mu}\left(\sigma^{\mu}\right)\right\| \geq \frac{1}{\mu}\left(\left\|\sigma^{\mu}\right\|-\left\|J_{\mathbf{M}}^{\mu}\left(\sigma^{\mu}\right)\right\|\right) \\
& \geq \frac{1}{\mu}\left(\left\|\sigma^{\mu}\right\|-\left\|\sigma^{\mu}-\mu \varepsilon \frac{\sigma^{\mu}}{\left\|\sigma^{\mu}\right\|}\right\|\right) \\
& =\frac{1}{\mu}\left(\left\|\sigma^{\mu}\right\|-\left(1-\frac{\mu \varepsilon}{\left\|\sigma^{\mu}\right\|}\right)\left\|\sigma^{\mu}\right\|\right)=\varepsilon
\end{aligned}
$$

Previous developments show us that it is sufficient to consider only the case when the sliding variable $\sigma^{\mu} \in \varepsilon \mu \mathbb{B}_{m}$, (since for the case $\sigma^{\mu} \notin \varepsilon \mu \mathbb{B}_{m}$ we have already shown that (35) is strictly negative). Hence, letting $\left\|\sigma^{\mu}\right\| \leq \mu \varepsilon$, and recalling that in this case $J_{\mathbf{M}}^{\mu}\left(\sigma^{\mu}\right)=0$, it follows that $\mathcal{M}^{\mu}\left(\sigma^{\mu}\right)=\frac{1}{\mu} \sigma^{\mu}$, and (35) transforms into

$$
\begin{aligned}
\dot{V} & \leq-\lambda_{\min }(\tilde{Q})\left\|z^{\mu}\right\|^{2}-\left(\varepsilon \gamma\left(z^{\mu}\right)-W-\sqrt{\kappa}\left\|z^{\mu}\right\|\right)\left\|\sigma^{\mu}\right\|-\gamma\left(z^{\mu}\right)\left\|\sigma^{\mu}\right\|\left(\frac{\left\|\sigma^{\mu}\right\|}{\mu}-\varepsilon\right) \\
& \leq-\lambda_{\min }(\tilde{Q})\left\|z^{\mu}\right\|^{2}-\left(\varepsilon \gamma\left(z^{\mu}\right)-W-\sqrt{\kappa}\left\|z^{\mu}\right\|\right)\left\|\sigma^{\mu}\right\|-\gamma\left(z^{\mu}\right) \frac{\left\|\sigma^{\mu}\right\|^{2}}{\mu}+\gamma\left(z^{\mu}\right) \varepsilon^{2} \mu .
\end{aligned}
$$

Let $L_{c}=\left\{z^{\mu} \in \mathbb{R}^{n} \mid V\left(z^{\mu}\right) \leq c,\right\}$ be the level sets of the function $V$ and let $c>0$, be such that the initial condition $z_{0} \in L_{c}$ and $r \mathbb{B}_{n} \subset L_{c}$ for some $r>0$. Then $\gamma(\cdot)$ is uniformly bounded in $L_{c}$ by some $\bar{\gamma}>0$, and for any $z \in L_{c} \backslash r \mathbb{B}_{n}$ we have that

$$
\dot{V} \leq-\left(\lambda_{\min }(\tilde{Q})-\frac{\bar{\gamma} \varepsilon^{2} \mu}{r^{2}}\right)\left\|z^{\mu}\right\|^{2}-\left(\varepsilon \gamma\left(z^{\mu}\right)-W-\sqrt{\kappa}\left\|z^{\mu}\right\|\right)\left\|\sigma^{\mu}\right\|-\gamma\left(z^{\mu}\right) \frac{\left\|\sigma^{\mu}\right\|^{2}}{\mu} .
$$

From (36) we can conclude that for all $\mu>0$ small enough such that

$$
\mu<\frac{r^{2} \lambda_{\min }(\tilde{Q})}{\varepsilon^{2} \bar{\gamma}}=: \mu^{*}
$$

the set $L_{c}$ is positively invariant, (since $\dot{V}<0$ in bd $\left(L_{c}\right)$ ), and boundedness of the trajectories in the time interval $[0, T]$ follows. A classical argument by contradiction gives us the existence of solutions of (26) for all $T>0$. It remains to show that for any $z^{\mu}(0)=z(0)=z_{0} \in \mathbb{R}^{n}$, the sequences $\left\{z^{\mu}\right\}_{\mu>0}$ formed by the solutions of (26) converge to a solution of (13),(22) as $\mu \downarrow 0$. Continuing with the proof, let $z_{0}^{\mu} \in \mathbb{R}^{n}$ be fixed, then there exists a $c>0$ such that $z^{\mu}(0) \in L_{c}$, and we have that any solution of $(26)$ satisfies $z^{\mu} \in C\left([0, T] ; \mathbb{R}^{n}\right)$ for any $T>0$, where $C\left([0, T] ; \mathbb{R}^{n}\right)$ refers to the Banach space of continuous functions from $[0, T]$ to $\mathbb{R}^{n}$ with norm $\|y\|=\sup _{t \in[0, T]}\|y(t)\|$. Further, the sequence of trajectories $\left\{z^{\mu}\right\}_{\mu>0}$ is uniformly bounded for all $0<\mu<\mu^{*}$ where $\mu^{*}$ satisfies (37) (since the set $L_{c}$ is positively invariant). On the other hand, from the assumption that the domain of $\mathbf{M}$ is all $\mathbb{R}^{m}$ it follows that $\mathcal{M}^{\mu}\left(\sigma^{\mu}(t)\right)$ is uniformly bounded. Actually, from the fact that the set $L_{c}$ is a compact subset of $\mathbb{R}^{n}$, it follows that there exist a compact subset $\tilde{L}_{c} \subset \mathbb{R}^{m}$, such that $\sigma^{\mu}(t) \in \tilde{L}_{c}$ for all $t \geq 0$ and all $0<\mu<\mu^{*}$, and a finite collection of open sets $\left\{O_{i}\right\} \subset \mathbb{R}^{m}$ such that:

1. $\tilde{L}_{c} \subset \cup_{i=1}^{r} O_{i}$,

2. For each $i \in\{1, \ldots, r\}, \mathbf{M}\left(O_{i}\right) \subset b_{i} \mathbb{B}_{m}$, for some $0<b_{i}<+\infty$.

Consequently, $\mathbf{M}\left(\sigma^{\mu}(t)\right) \subset \cup_{i=1}^{r} \mathbf{M}\left(O_{i}\right) \subset \max _{i \in\{1, \ldots, r\}} b_{i} \mathbb{B}_{m}$. Hence, invoking (2) it follows that $\left\|\mathcal{M}^{\mu}\left(\sigma^{\mu}(t)\right)\right\| \leq\left\|\operatorname{Proj}_{\mathbf{M}\left(\sigma^{\mu}(t)\right)}(0)\right\| \leq \max _{i \in\{1, \ldots, r\}} b_{i}$. Therefore, from Assumption 3, together with (26) and the conclusion about the boundedness of its solutions it follows that for any $0<\mu<\mu^{*}, \dot{z}^{\mu} \in$ $\mathcal{L}_{\infty}\left([0, T] ; \mathbb{R}^{n}\right)$ is uniformly bounded. Hence, we have that the sequence $\left\{z^{\mu}\right\}_{\mu>0}$ is equicontinuous. By a direct application of the Arzelà-Ascoli Theorem [29, Theorem 1.3.8] we get that there exists a subsequence (still denoted by) $\left\{z^{\mu}\right\}_{\mu>0}$ such that $z^{\mu} \rightarrow z$ for some $z \in C\left([0, T] ; \mathbb{R}^{n}\right.$ ) uniformly 
in $[0, T]$. On the other hand, because $\dot{z}^{\mu} \in \mathcal{L}_{\infty}\left([0, T] ; \mathbb{R}^{n}\right)$, an application of the Banach-Alaoglu Theorem [29, Theorem 2.4.3] gives us that there exists a function $q \in \mathcal{L}_{\infty}\left([0, T] ; \mathbb{R}^{n}\right)$, such that $\dot{z}^{\mu} \rightarrow q$ in the weak* topology, i.e.,

$$
\lim _{\mu \downarrow 0} \int_{0}^{T}\left\langle\dot{z}^{\mu}(t)-q(t), s(t)\right\rangle d t=0, \text { for all } s \in \mathcal{L}_{1}\left([0, T] ; \mathbb{R}^{n}\right) .
$$

Moreover, from the fact that $z(t)=z(0)+\int_{0}^{T} q(t) d t$ we infer that $q=\dot{z}$ almost everywhere. Notice that since the considered time domain is bounded, we have that $\mathcal{L}_{2}\left([0, T] ; \mathbb{R}^{n}\right) \subset \mathcal{L}_{1}\left([0, T] ; \mathbb{R}^{n}\right)$ $[28$, Corollary 1, Chapter VIII $]$. Hence, $\dot{z}^{\mu}$ converges weakly in $\mathcal{L}_{2}\left([0, T] ; \mathbb{R}^{n}\right)$. From the continuity assumption of $\hat{\Delta}_{A}$ and the convergence of $z^{\mu}$ and $\dot{z}^{\mu}$ to $z$ and $\dot{z}$ respectively, it becomes clear that $z$ satisfies (13a). In fact,

$$
\begin{aligned}
\dot{z}_{1}^{\mu}=B_{\perp}^{\top}\left(A+\hat{\Delta}_{A}\left(t, z^{\mu}\right)\right) P B_{\perp}\left(B_{\perp}^{\top} P B_{\perp}\right)^{-1} z_{1}^{\mu}+B_{\perp}^{\top}\left(A+\Delta_{A}\left(t, z^{\mu}\right)\right) B \sigma^{\mu} \rightarrow \\
B_{\perp}^{\top}\left(A+\hat{\Delta}_{A}(t, z)\right) P B_{\perp}\left(B_{\perp}^{\top} P B_{\perp}\right)^{-1} z+B_{\perp}^{\top}\left(A+\Delta_{A}(t, z)\right) B \sigma=\dot{z}_{1} .
\end{aligned}
$$

Additionally, setting $\theta^{\mu}:=\dot{\sigma}^{\mu}+K \sigma^{\mu}-\hat{w}-\hat{\phi}_{m}$ we have that for any $\varphi \in \mathcal{L}_{2}\left([0, T] ; \mathbb{R}^{m}\right)$,

$$
\begin{aligned}
& \int_{0}^{T}\left\langle\frac{\theta^{\mu}(t)}{\gamma\left(z^{\mu}(t)\right)}-\frac{\theta(t)}{\gamma(z(t))}, \varphi(t)\right\rangle d t \\
& \quad=\int_{0}^{T}\left(\frac{1}{\gamma\left(z^{\mu}(t)\right)}-\frac{1}{\gamma(z(t))}\right)\left\langle\theta^{\mu}(t), \varphi(t)\right\rangle d t+\int_{0}^{T}\left\langle\frac{\theta^{\mu}(t)-\theta(t)}{\gamma(z(t))}, \varphi(t)\right\rangle d t
\end{aligned}
$$

From (25) if follows that $\gamma(z)>\frac{\rho}{\varepsilon}$ for any $z \in \mathbb{R}^{n}$. Thus, there exists a $\mu>0$, such that for all $\mu \leq \mu^{*}$, we have,

$$
\begin{aligned}
\int_{0}^{T}\left\langle\frac{\theta^{\mu}(t)}{\gamma\left(z^{\mu}(t)\right)}-\right. & \left.\frac{\theta(t)}{\gamma(z(t))}, \varphi(t)\right\rangle d t \\
& \leq \int_{0}^{T} \frac{\varepsilon^{2}}{\rho^{2}} L_{\gamma}\left\|z^{\mu}(t)-z(t)\right\|\left\|\theta^{\mu}(t)\right\|\|\varphi(t)\| d t+\int_{0}^{T} \frac{\varepsilon}{\rho}\left\langle\theta^{\mu}(t)-\theta(t), \varphi(t)\right\rangle d t,
\end{aligned}
$$

where $L_{\gamma}>0$ refers to the Lipschitz constant of the function $\gamma$. Hence:

$$
\zeta^{\mu}:=\frac{\dot{\sigma}^{\mu}+K \sigma^{\mu}-\hat{w}\left(t, z^{\mu}\right)-\hat{\phi}_{m}\left(t, z^{\mu}\right)}{\gamma\left(z^{\mu}\right)} \rightarrow \frac{\dot{\sigma}+K \sigma-\hat{w}(t, z)-\hat{\phi}_{m}(t, z)}{\gamma(z)}=: \zeta, \text { as } \mu \downarrow 0,
$$

weakly in $\mathcal{L}_{2}\left([0, T] ; \mathbb{R}^{m}\right)$ for any $T>0$. Finally, from $[4$, p. 146] it follows that the set-valued map $\mathbf{M}$ seen as a set-valued map from $\mathcal{L}_{2}\left([0, T], \mathbb{R}^{m}\right)$ to the subsets of $\mathcal{L}_{2}\left([0, T], \mathbb{R}^{m}\right)$ is also maximal monotone. Thus, since $J_{\mathbf{M}}^{\mu}\left(\sigma^{\mu}\right) \rightarrow \sigma$ uniformly in $C\left([0, T], \mathbb{R}^{m}\right)$, [4, p.144] (and consequently strongly in $\left.\mathcal{L}_{2}\left([0, T] ; \mathbb{R}^{m}\right)\right)$, the left-hand side of $(39)$ is equal to $\zeta^{\mu}=\mathcal{M}^{\mu}\left(\sigma^{\mu}\right)$ and $\mathcal{M}^{\mu}\left(\sigma^{\mu}\right) \in \mathbf{M}\left(J_{\mathbf{M}}^{\mu}\left(\sigma^{\mu}\right)\right)$, [4, p. 144]. Invoking Proposition 1 in Section 2 allows us to conclude that $\zeta \in \mathbf{M}(\sigma)$, that is, the differential inclusion (13),(22) is satisfied. This finishes the proof.

Remark 3. Notice that the assumption Dom $\mathbf{M}=\mathbb{R}^{m}$ rules out multivalued controllers with compact domain as those introduced in Example 3. However, the use of set-valued maps whose domain is not all $\mathbb{R}^{m}$ is possible using $\gamma>0$ constant, since we fall in the case of differential inclusion with maximal monotone right-hand side, see e.g., [9, 15].

Theorem 2. Let the assumptions of Theorem 1 hold. Then, the subsystem (13b) with set-valued controller (22) is globally finite-time Lyapunov stable whenever,

$$
\varepsilon \gamma(z) \geq \rho+W+\sqrt{\kappa}\|z\|
$$

where $\varepsilon$ is given in (24), and $\rho>0$ is an arbitrary constant. 
Proof. We consider the positive definite function of $\sigma, V(\sigma)=\frac{1}{2} \sigma^{\top} \sigma$. From the proof of Theorem 1 we have that $z_{1}$ is bounded. So, differentiating $V$ along the trajectories of (13b) results in $\dot{V}=$ $\sigma^{\top} \dot{\sigma}=\sigma^{\top}\left(u^{\mathrm{sv}}+w+\phi_{m}\right)$. From (22) there exists a $\zeta \in \mathbf{M}(\sigma)$ such that $u^{\mathrm{sv}}=-K \sigma-\gamma(x) \zeta$ and then,

$$
\begin{aligned}
\dot{V} & \leq-\sigma^{\top} K \sigma-\gamma(z) \sigma^{\top} \zeta+\left\|w+\phi_{m}\right\|\|\sigma\| \\
& \leq-(\varepsilon \gamma(z)-W-\sqrt{\kappa}\|z\|)\|\sigma\|,
\end{aligned}
$$

where we have used (24) and the fact that $K>0$. Hence, if (40) holds, then $\dot{V}<-\rho\|\sigma\|$. Finally, after integration of both sides of the last inequality an upper-bound for the time $t^{*}$ such that $\sigma(t)=0$ for all $t \geq t^{*}$, is obtained as: $t^{*} \leq \sqrt{2 V(0)} / \rho$.

It is worth to mention that Theorem 2 does not make mention to the uniqueness of solutions, but we have proved instead that all the solutions converge to the sliding surface. The next step consists in showing the asymptotic stability of the whole system (13),(22).

Theorem 3. Let the assumptions of Theorem 1 hold. Then, the closed-loop system (13), (22) is globally asymptotically stable.

Proof. Consider the Lyapunov-function candidate,

$$
V\left(z_{1}, \sigma\right):=\frac{1}{2} z_{1}^{\top}\left(B_{\perp}^{\top} P B_{\perp}\right)^{-1} z_{1}+\frac{1}{2} \sigma^{\top} \sigma .
$$

Let $\zeta$ be an element in $\mathbf{M}(\sigma)$, differentiating (41) along the system's trajectories yields

$$
\begin{aligned}
\dot{V} & \leq-\lambda_{\min }(\tilde{Q})\|z\|^{2}+\sigma^{\top}\left(-\gamma(z) \zeta+\hat{w}(t, z)+\hat{\phi}_{m}(t, z)\right) \\
& \leq-\lambda_{\min }(\tilde{Q})\|z\|^{2}-(\varepsilon \gamma(z)-(W+\sqrt{\kappa}\|z\|))\|\sigma\|<0,
\end{aligned}
$$

where the matrix $\tilde{Q}=\tilde{Q}^{\top}>0$ is defined in (33) and we made use of (24). This concludes the proof.

An important case arises when we ask for a constant gain $\gamma>0$. In this case the existence of solutions has been deeply studied (see, e.g., [9], [15], [19]) and from the practical point of view, we sacrifice the global stability for semi-global stability and the uniqueness of solutions is retrieved.

Corollary 1. Let the Assumptions 1-6 hold, let $\alpha>0, \delta>0$ and $P=P^{\top}$ be such that (5), (20) hold, and let $L_{c} \subset \mathbb{R}^{n}$ be a compact set specified below in the proof. Then, for each initial condition that satisfies $\left(z_{1}(0), \sigma(0)\right) \in L_{c}$, for some $c>0$, the closed-loop system (13) with set-valued controller

$$
-u^{\mathrm{sv}} \in K \sigma+\gamma \mathbf{M}(\sigma)
$$

where $K=K^{\top}>0$ satisfies (19), is semi-globally asymptotically stable whenever

$$
\varepsilon \gamma \geq \beta+W+\sqrt{\kappa} \max _{z \in L_{c}}\{\|z\|\}
$$

where $z=\left[z_{1}^{\top}, \sigma^{\top}\right]^{\top}, \kappa$ is given in (21), and $\beta>0$ is an arbitrary constant.

Proof. Consider the positive definite function $V\left(z_{1}, \sigma\right)$ as in (41) and let $L_{c}:=\left\{\left(z_{1}, \sigma\right) \in \mathbb{R}^{n} \mid V\left(z_{1}, \sigma\right) \leq\right.$ $c\}$ be the level sets of the function $V$. As first step we prove the positive invariance of the set $L_{c}$. To this end we take the time derivative of $V$ along the system trajectories, yielding again (42) changing $\gamma(z)$ by $\gamma$. Hence, in the light of (44), we can conclude that $\dot{V}<0$ for all $\sigma \in \operatorname{bd}\left(L_{c}\right)$ and the positive invariance follows. Now, let $\left(z_{1}(0), \sigma(0)\right) \in L_{c}$ for some $c>0$, then from (42) and the fact that the maximum in (44) is attained in the boundary of $L_{c}$ it follows that $\dot{V}<0$ and we arrive at the conclusion. 
From Corollary 1 it follows that the multivalued controller (43) carries the system (13) into the sliding surface $\sigma=0$ in finite time. Moreover, as a consequence of the maximal monotonicity of the set-valued map $\gamma \mathbf{M}(\cdot)$ we have uniqueness of solutions of the closed-loop system (13), (43). Indeed, consider the following differential inclusion

$$
\dot{z} \in f(t, z)-\gamma \mathbf{N}(z)
$$

where:

$$
\begin{aligned}
f(t, z)=\left[\begin{array}{cc}
B_{\perp}^{\top}\left(A+\hat{\Delta}_{A}(t, z)\right) P B_{\perp}\left(B_{\perp}^{\top} P B_{\perp}\right)^{-1} & B_{\perp}^{\top}\left(A+\hat{\Delta}_{A}(t, z)\right) B \\
0 & -K
\end{array}\right]\left[\begin{array}{c}
z_{1} \\
\sigma
\end{array}\right] & +\left[\begin{array}{c}
0 \\
\hat{w}(t, z)+\hat{\phi}_{m}(t, z)
\end{array}\right]
\end{aligned}
$$

is a locally Lipschitz function in its second argument and $\mathbf{N}: \mathbb{R}^{n} \rightrightarrows \mathbb{R}^{n}$ is a maximal monotone set-valued map described by $z \mapsto\left[0, \zeta^{\top}\right]^{\top}$ and $\zeta \in \mathbf{M}(\sigma)$. Then, assuming that there exist two solutions of (45) denoted by $z^{1}$ and $z^{2}$, it follows that,

$$
\begin{aligned}
\frac{d}{d t} \frac{1}{2}\left\|z^{1}-z^{2}\right\|^{2} & =\left\langle\dot{z}^{1}-\dot{z}^{2}, z^{1}-z^{2}\right\rangle \\
& =\left\langle f\left(t, z^{1}\right)-f\left(t, z^{2}\right), z^{1}-z^{2}\right\rangle-\gamma\left\langle\eta^{1}-\eta^{2}, z^{1}-z^{2}\right\rangle \\
& \leq L_{f}\left\|z^{1}-z^{2}\right\|^{2},
\end{aligned}
$$

where $\eta^{i} \in \mathbf{N}\left(z^{i}\right), i=1,2$ and $L_{f}$ refers to the Lipschitz constant of the function $f$. The application of Gronwall's inequality leads to

$$
\left\|z^{1}(t)-z^{2}(t)\right\| \leq\left\|z^{1}(0)-z^{2}(0)\right\| e^{L_{f} t},
$$

for all $t \geq 0$, making evident the uniqueness of solutions.

It is a well known fact that in the continuous-time setting the selection of the values that maintain the sliding regime depends explicitly on the values of the disturbances $\hat{w}$ and $\hat{\phi}_{m}$ which are by definition unknown. For that reason, in practical applications it is common to use a regularized version of the controller (22), leading us to the concept of boundary layer control [42]. In general, the regularization is made in an arbitrary way. In our context the regularization is well defined through the use of the Yosida regularization and as was shown in the proof of Theorem 1 this approach leads to trajectories that are in a neighbourhood of one solution of the differential inclusion (13). In the sequel we present an example for the case of the unitary vector approach.

Consider the set-valued map $\mathbf{M}$ as in the Example 2 and a constant gain $\gamma>0$. From the proof of Theorem 1, it follows that our regularized control is given by the maximal monotone single valued map $\mathcal{M}^{\mu}$, which in this case is given by

$$
\mathcal{M}^{\mu}(\sigma)=\nabla f^{\mu}(\sigma)=\frac{1}{\mu}\left(\sigma-\operatorname{Prox}_{\mu f}(\sigma)\right)= \begin{cases}\frac{\sigma}{\|\sigma\|}, & \text { if }\|\sigma\|>\mu, \\ \frac{1}{\mu} \sigma, & \text { otherwise. }\end{cases}
$$

It is worth to mention that (46) differs from the commonly used regularization $\frac{\sigma}{\|\sigma\|+\rho}$, with $\rho>0$ sufficiently small. Therefore, in the maximal monotone approach we have a unique way of computing the regularized controller coming from a set-valued maximal monotone map leading to a closed-loop system whose trajectories converge into a neighborhood of the origin. In the next section we shall study the design of this kind of maximal monotone controllers in the discrete-time setting.

\section{Design of discrete-time sliding-mode controllers using max- imal monotone maps}

In this section we present a methodology for the digital implementation of discrete-time sliding mode controllers using maximal monotone maps. The design process is revisited step-by-step in order to 
show how the implicit discrete-time scheme proposed in $[1,2]$ allows us to make a proper selection of the values of the control input at each sampling instant, and consequently reduces drastically the chattering effect at higher sampling rates.

\subsection{The plant representation}

We start considering the discrete-time model of (4) through the use of the Euler's method, i.e., we take a constant sampling time $t_{k+1}-t_{k}=h>0$ for all $k \geq 0$, and we obtain,

$$
x_{k+1}=\left(I_{n}+h A\right) x_{k}+h B\left(u_{k}+w_{k}\right)+h \Delta_{A} x_{k} .
$$

It is worth to mention that in the absence of the parametric disturbances $\left(\Delta_{A}(t, x) \equiv 0\right)$, the system (47) becomes linear and the ZOH (Zero-Order Hold) method can be applied in order to obtain the equations of the dynamics in discrete time. Using the $\mathrm{ZOH}$ method has the disadvantage that for big sampling times, the resulting discrete-time system could result in an uncontrollable system [27]. This disadvantage is not present when the Euler's method is applied. Namely, assume that for a linear (unperturbed) continuous-time system the pair $(A, B)$ is controllable, i.e., $\operatorname{rank}\left(\left[\lambda I_{n}+A \mid B\right]\right)=n$ for all $\lambda \in \sigma(A)$. Then, after applying the Euler's method, the system matrices become $\left(I_{n}+h A, h B\right)$. The condition for controllability of this new pair translates into, $\operatorname{rank}\left[\mu I_{n}-\left(I_{n}+h A\right) \mid h B\right]=n$ for all $\mu \in \sigma\left(I_{n}+h A\right)$, which is trivially satisfied in the light of $\mu_{i}=1+h a \lambda_{i}$, for all $i=1, \ldots, n$ and the assumed controllability of the original continuous-time system. Previous lines shows that using the Euler's discretization method leads to controllable systems but unfortunately we lose the exact representation of the discrete-time dynamics and also it is not possible to obtain an arbitrary desired decaying rate $a$ (see Proposition 4) which in our context translates into considering high sampling rates for the domination of the unmatched disturbance. Along all this section we also consider that Assumptions 1 through 6 hold. In the discrete-time context the counterpart of Proposition 4 is given as:

Proposition 7. Assumption 1 implies that for some $a>0$ such that $0<2 h a<1$, there exists a symmetric positive definite matrix $X \in \mathbb{R}^{n \times n}$ satisfying the matrix inequality:

$$
B_{\perp}^{\top}\left(A X+X A^{\top}+2 a X\right) B_{\perp}+h B_{\perp}^{\top}\left(X A^{\top} B_{\perp}\left(B_{\perp}^{\top} X B_{\perp}\right)^{-1} B_{\perp}^{\top} A X\right) B_{\perp}<0 .
$$

Proof. Stabilizability of the system (47) is equivalent to the existence of a matrix $K \in \mathbb{R}^{m \times n}$ such that for any $2 h a \in(0,1)$, there exists a matrix, $U \in \mathbb{R}^{n \times n}, U=U^{\top}>0$ satisfying the discrete-time Lyapunov's equation:

$$
(1-2 h a) U-(I+h A-h B K)^{\top} U(I+h A-h B K)>0 .
$$

Pre and post multiplying by $U^{-1}$ and setting $W=K U^{-1}$ yields,

$$
-h\left(2 a U^{-1}+A U^{-1}+U^{-1} A^{\top}-B W-W^{\top} B^{\top}\right)-h^{2}\left(A U^{-1}-B W\right)^{\top} U\left(A U^{-1}-B W\right)>0 .
$$

Hence, applying the Schur's complement formula we obtain the LMI

$$
\left[\begin{array}{cc}
-h\left(2 a U^{-1}+A U^{-1}+U^{-1} A^{\top}-B W-W^{\top} B^{\top}\right) & h\left(U^{-1} A^{\top}-W^{\top} B\right) \\
h\left(A U^{-1}-B W\right) & U^{-1}
\end{array}\right]>0 .
$$

Recalling that $B_{\perp} \in \mathbb{R}^{n \times(n-m)}$ has full column rank, it follows that the previous inequality implies

$$
\left[\begin{array}{cc}
-h B_{\perp}^{\top}\left(2 a U^{-1}+A U^{-1}+U^{-1} A^{\top}\right) B_{\perp} & h B_{\perp}^{\top} U^{-1} A^{\top} B_{\perp} \\
h B_{\perp}^{\top} A U^{-1} B_{\perp} & B_{\perp}^{\top} U^{-1} B_{\perp}
\end{array}\right]>0,
$$

where we have applied the full row rank congruence transformation

$$
\left[\begin{array}{cc}
B_{\perp}^{\top} & 0_{n-m \times n} \\
0_{n \times n-m} & B_{\perp}^{\top}
\end{array}\right] \in \mathbb{R}^{2(n-m) \times 2 n} .
$$

Finally, applying once again the Schur's complement formula to (49) and setting $X=U^{-1}$ we obtain the desired result. 
From (48) it is easy to see that as $h \downarrow 0$ the solution of the matrix inequality (48) approaches the solution of the LMI (5).

To finish this subsection we compute a bound for $\Delta_{A}$ that will be useful in the forthcoming sections.

Proposition 8. Let $X=X^{\top}>0$ be such that

$$
X-I_{n}>0
$$

then,

$$
\Lambda^{-1}-\Delta_{A}^{\top} B_{\perp}\left(B_{\perp}^{\top} X B_{\perp}\right)^{-1} B_{\perp}^{\top} \Delta_{A}>0 .
$$

Proof. From Assumption 3 together with the bound on $X$ imposed by (50) it follows that $\Delta_{A} \Lambda \Delta_{A}^{\top}<$ $X$. Since $B_{\perp}$ has full column rank, it follows that $B_{\perp}^{\top} X B_{\perp}-B_{\perp}^{\top} \Delta_{A} \Lambda \Delta_{A}^{\top} B_{\perp}>0$. Using the Schur's complement formula we obtain,

$$
\left[\begin{array}{cc}
B_{\perp}^{\top} X B_{\perp} & B_{\perp}^{\top} \Delta_{A} \\
\Delta_{A}^{\top} B_{\perp} & \Lambda^{-1}
\end{array}\right]>0
$$

and applying once again the Schur's complement formula we obtain the desired result.

In the sequel we will assume that $X$ satisfies (48) together with (50) and consequently (51) also holds.

\subsection{Design of the sliding surface}

In this subsection the methodology for the design of the sliding surface mimics its continuous counterpart. First, we start with a sliding manifold of the form $\sigma_{k}=S x_{k}$ and conditions on the matrix $S$ are derived. In fact, it is shown that the resulting hyperplane has the same structure as its continuous-time analog $C$. We make the following assumption,

Assumption 7. The product $S B$ is nonsingular.

Analogous to the continuous-time context, we start computing the equivalent control in order to see how the disturbance affects the sliding regime. In the discrete-time case, the necessary sliding condition $\dot{\sigma}=0$ is transformed into the fixed-point condition $\sigma_{k+1}=\sigma_{k}$ from which we obtain the equivalent control as ${ }^{1}$ :

$$
u_{k}^{\mathrm{eq}}=\frac{1}{h}(S B)^{-1}\left(\sigma_{k}-S\left(I_{n}+h A\right) x_{k}-h S \Delta_{A} x_{k}\right)-w_{k}
$$

Notice that the fixed-point condition $\sigma_{k+1}=\sigma_{k}$ is usually neglected and changed for the condition $\sigma_{k+1}=0$. We will see that the fixed-point condition is well fitted for the estimation of the control law that will achieve the sliding motion. The equivalent closed-loop dynamics in sliding mode results in:

$$
x_{k+1}^{\mathrm{eq}}=\left(I_{n}-B(S B)^{-1} S\right)\left(I_{n}+h A\right) x_{k}^{\mathrm{eq}}+B(S B)^{-1} \sigma_{k}+h\left(I_{n}-B(S B)^{-1} S\right) \Delta_{A} x_{k} .
$$

From (53) it becomes clear that the structure of the sliding surface will be the same as in the continuous-time framework, i.e., throughout this section we set $S=\left(B^{\top} X^{-1} B\right)^{-1} B^{\top} X^{-1}$. Notice that the both surfaces $(C$ and $S$ ) are not exactly the same since $P$ satisfies (5) and $X$ satisfies (48) instead, but $S$ tends to $C$ as $h$ decreases to zero.

\footnotetext{
${ }^{1}$ As alluded to above, what we call the equivalent control here, is not the same as what is called the equivalent control in [24].
} 


\subsection{Controller design}

In this subsection we follow the discrete version of the two-steps design methodology used in the previous section. The main difference with the continuous part relies on the discretization scheme used for the control $u^{\mathrm{sv}}$. It is shown that the implicit discretization approach inherits the robustness provided by the maximal monotone operators presented in Section 3. The first step consists in computing the nominal control using the fixed-point condition $\sigma_{k+1}=\sigma_{k}$ leading to

$$
u_{k}^{\text {nom }}=\frac{1}{h}(S B)^{-1}\left(\sigma_{k}-S\left(I_{n}+h A\right) x_{k}\right) .
$$

Substitution of (54) into the discrete-time dynamics (47) yields

$$
x_{k+1}=\left(I_{n}-B(S B)^{-1} S\right)\left(I_{n}+h A\right) x_{k}+B(S B)^{-1} \sigma_{k}+h B\left(u_{k}^{\mathrm{sv}}+w_{k}\right)+h \Delta_{A} x_{k} .
$$

Consider the coordinates transformation $z_{k}=T x_{k}$ with $T$ given in (11) but changing the matrix $P$ by its discrete-time counterpart $X$. Hence, after simple computations we get the closed-loop system in regular form,

$$
\left\{\begin{array}{l}
z_{k+1}^{1}=B_{\perp}^{\top}\left(I_{n}+h A+h \Delta_{A}\right) X B_{\perp}\left(B_{\perp}^{\top} X B_{\perp}\right)^{-1} z_{k}^{1}+B_{\perp}^{\top}\left(I_{n}+h A+h \Delta_{A}\right) B \sigma_{k} \\
\sigma_{k+1}=\sigma_{k}+h\left(u_{k}^{\mathrm{sv}}+w_{k}+\eta_{k}^{m}\right)
\end{array}\right.
$$

where the term $\eta_{k}^{m}$ refers to the matched part of the disturbance $\Delta_{A} x_{k}$, i.e., $\eta_{k}^{m}=S \Delta_{A} T^{-1} z_{k}=$ $\left(B^{\top} X^{-1} B\right)^{-1} B^{\top} X^{-1} \Delta_{A} T^{-1} z_{k}$, see Remark 1. It is noteworthy that system (56) is the discretetime counterpart of (13). It is clear that the disturbance term $\eta_{k}^{m}$ satisfies a linear growth condition similar to that associated with the term $\phi_{m}$. Thus the following holds.

Proposition 9. The disturbance term $\eta_{k}^{m}$ satisfies the linear growth condition $\left\|\eta_{k}^{m}\right\| \leq \sqrt{\bar{\kappa}}\left\|z_{k}\right\|$, where

$$
\bar{\kappa}:=\frac{\lambda_{\max }(X) \lambda_{\max }\left(\Lambda^{-1}\right)}{\lambda_{\min }\left(B^{\top} X^{-1} B\right) \lambda_{\min }(X)} \max \left\{\frac{1}{\lambda_{\min }\left(B_{\perp}^{\top} X B_{\perp}\right)}, \lambda_{\max }\left(B^{\top} X^{-1} B\right)\right\} .
$$

\subsubsection{The set-valued controller}

We continue with the design of the multivalued part of the controller. The main difference with the continuous-time part relies here, where, because of the discretization method employed, it is possible to make a selection for the values of the controller that will compensate for the disturbances that affect the resulting closed-loop system. Specifically, we use the implicit Euler's method and we show how the system automatically makes the selection of the values that will compensate for the disturbance. As a motivation of the implicit scheme used, we study first the following equivalent controller,

$$
-u_{k}^{\mathrm{sv}} \in \gamma \mathbf{M}\left(\sigma_{k+1}\right)
$$

where $\gamma>0$ is considered constant. Two important questions arise: is the proposed set-valued controller (58) non-anticipative? and why is it called equivalent? The label equivalent is because, in the sliding phase, $u_{k}^{\mathrm{sv}}$ is equal to $u_{k}^{\mathrm{eq}}-u_{k}^{\mathrm{nom}}$, i.e., the control action $u_{k}=u_{k}^{\mathrm{nom}}+u_{k}^{\mathrm{sv}}$, with $u_{k}^{\mathrm{sv}}$ satisfying (58), coincides with the equivalent control (52). Indeed, consider the closed-loop system (56b), (58). It follows that,

$$
\begin{aligned}
& \sigma_{k}-\sigma_{k+1}+h\left(w_{k}+\eta_{k}\right) \in h \gamma \mathbf{M}\left(\sigma_{k+1}\right) \Leftrightarrow \quad \sigma_{k}+h\left(w_{k}+\eta_{k}\right) \in(I+h \gamma \mathbf{M})\left(\sigma_{k+1}\right) \\
& \Leftrightarrow \quad \sigma_{k+1}=(I+h \gamma \mathbf{M})^{-1}\left(\sigma_{k}+h\left(w_{k}+\eta_{k}\right)\right) \\
& \Leftrightarrow \quad \sigma_{k+1}=J_{\gamma \mathbf{M}}^{h}\left(\sigma_{k}+h\left(w_{k}+\eta_{k}\right)\right),
\end{aligned}
$$

where $J_{\gamma \mathbf{M}}^{h}$ refers to the resolvent of the maximal monotone map $\gamma \mathbf{M}$ of index $h$. Hence, the discretetime closed-loop dynamics of the sliding variable results in the difference equation (59). An explicit expression for the controller is obtained after substitution of (59) into (56b) as

$$
u_{k}^{\mathrm{sv}}=-\frac{1}{h}\left(I-J_{\gamma \mathbf{M}}^{h}\right)\left(\sigma_{k}+h\left(w_{k}+\eta_{k}^{m}\right)\right)=-\mathcal{M}_{\gamma}^{h}\left(\sigma_{k}+h\left(w_{k}+\eta_{k}^{m}\right)\right) .
$$


where the map $\mathcal{M}_{\gamma}^{h}$ refers to the Yosida approximation of the set-valued map $\gamma \mathbf{M}$ of index $h$. At this point it is worth to mention that the selection process was done automatically by the system, i.e., the closed-loop system selects one and only one input from the maximal monotone map $\mathbf{M}$ in order to compensate for the disturbance term $w_{k}+\eta_{k}^{m}$. Thus, in ideal sliding mode $\sigma_{k+1}=\sigma_{k}=0$ implies $u_{k}^{\mathrm{sv}}=-\frac{1}{h}\left(I-J_{\gamma \mathbf{M}}^{h}\right)\left(h\left(w_{k}+\eta_{k}^{m}\right)\right)$. Now, assuming that $w_{k}+\eta_{k}^{m} \in \gamma \mathbf{M}(0)$ it follows that $u_{k}^{\mathrm{sv}}=-w_{k}-\eta_{k}^{m}$, (since $J_{\gamma \mathbf{M}}^{h}(w)=0$ for all $\left.w \in \gamma \mathbf{M}(0)\right)$. Therefore, $u_{k}=u_{k}^{\text {nom }}+u_{k}^{\mathrm{sv}}=u_{k}^{\mathrm{eq}}$. The previous development reveals that the implicit controller (58) makes sense.

Now we introduce the missing term $u_{k}^{\text {sv }}$ using an implicit approach, which has been studied theoretically in $[1,2,24]$ and tested experimentally in $[25,26,45]$ showing to be a very efficient way to deal with the chattering effect. It is clear that in a real implementation setting the selection procedure cannot be achieved directly, because if we try to mimic the same steps presented in the previous situation, we will have to impose the unreal assumption that we know perfectly the disturbance term $w_{k}+\eta_{k}^{m}$, see (60). Therefore some modification to the discrete-time controller (58) must be done. Roughly speaking, we consider the discrete-time scheme proposed in $[1,2,24]$ by creating a virtual nominal system from where the selection process is achieved. Next, the controller computed from the virtual nominal system is applied to the original discrete-time plant. Formally, instead of (56), (58), we consider the extended system,

$$
\left\{\begin{array}{l}
z_{k+1}^{1}=B_{\perp}^{\top}\left(I_{n}+h A+h \Delta_{A}\right) X B_{\perp}\left(B_{\perp}^{\top} X B_{\perp}\right)^{-1} z_{k}^{1}+B_{\perp}^{\top}\left(I_{n}+h A+h \Delta_{A}\right) B \sigma_{k} \\
\sigma_{k+1}=\tilde{\sigma}_{k+1}+h\left(w_{k}+\eta_{k}^{m}\right) \\
\tilde{\sigma}_{k+1}=\sigma_{k}+h u_{k}^{\mathrm{sv}} \\
-u_{k}^{\mathrm{sv}} \in K \tilde{\sigma}_{k+1}+\gamma \mathbf{M}\left(\tilde{\sigma}_{k+1}\right),
\end{array}\right.
$$

where $K \in \mathbb{R}^{m \times m}$ is a symmetric positive definite matrix specified below. System (61) represents the implementable discrete-time dynamics associated with the real continuous-time system (13). The variable $\tilde{\sigma}_{k+1}$ may be seen as the state of a nominal, undisturbed system, or as a dumb variable allowing to calculate the controller $u_{k}^{\mathrm{sv}}$. In this approach the control selection is made using the virtual undisturbed system (61c)-(61d), and the perturbation term is implicitly taken into account through the use of the real state $\sigma_{k}$ in (61c). Following the same steps as in (59), we have

$$
\begin{array}{rlrl}
\sigma_{k}-\tilde{\sigma}_{k+1} \in h K \tilde{\sigma}_{k+1}+h \gamma \mathbf{M}\left(\tilde{\sigma}_{k+1}\right) & \Leftrightarrow & & \sigma_{k} \in(I+h(K+\gamma \mathbf{M}))\left(\tilde{\sigma}_{k+1}\right) \\
& \Leftrightarrow & \tilde{\sigma}_{k+1}=(I+h(K+\gamma \mathbf{M}))^{-1}\left(\sigma_{k}\right) \\
& \Leftrightarrow & & \tilde{\sigma}_{k+1}=J_{\mathbf{N}}^{h}\left(\sigma_{k}\right),
\end{array}
$$

where $K=K^{\top}>0$ is an $m \times m$ matrix and the set-valued map $\mathbf{N}:=K+\gamma \mathbf{M}$ that maps $p \mapsto\left\{q \in \mathbb{R}^{m} \mid q=K p+\gamma \zeta, \zeta \in \mathbf{M}(p)\right\}$ is also maximal monotone [38, Exercise 12.4]. It follows from (61c) that the input selection applied to the system is explicitly given by

$$
u_{k}^{\mathrm{sv}}=-\frac{1}{h}\left(I-J_{\mathbf{N}}^{h}\right)\left(\sigma_{k}\right)=:-\mathcal{N}^{h}\left(\sigma_{k}\right),
$$

where $\mathcal{N}^{h}$ refers to the Yosida approximation of $\mathbf{N}$ of index $h$. Equation (63) shows the nonanticipation and the uniqueness of the control (61d) (since $\mathcal{N}^{h}$ is single valued). Hence, the discretetime closed-loop subsystem (61b)-(61d) is equivalent to,

$$
\left\{\begin{array}{l}
\sigma_{k+1}=\tilde{\sigma}_{k+1}+h\left(w_{k}+\eta_{k}^{m}\right), \\
\tilde{\sigma}_{k+1}=J_{\mathbf{N}}^{h}\left(\sigma_{k}\right) .
\end{array}\right.
$$

In this context the variable $\tilde{\sigma}_{k}$ is called the discrete sliding variable and when $\tilde{\sigma}_{k+n}=0$ for all $n \geq 1$ and some $k<+\infty$, we say that the system is in the discrete-time sliding phase [24].

\subsection{Stability of the closed-loop}

In this section the stability of the system (61) is proved. We start by computing the necessary conditions that the matrices $X$ and $K$ must satisfy under the assumption of ideal sliding phase, that is, 
$\sigma_{k}=0$. This step allows us to compare the discrete-time and the continuous-time approaches showing their similarities, and also providing some convergence results. To this end, we start considering the following discrete-time reduced order system:

$$
z_{k+1}^{1}=B_{\perp}^{\top}\left(I_{n}+h A+h \Delta_{A}\right) X B_{\perp}\left(B_{\perp}^{\top} X B_{\perp}\right)^{-1} z_{k}^{1},
$$

together with the Lyapunov-function candidate $V\left(z_{k}^{1}\right)=\frac{1}{2} z_{k}^{1 \top}\left(B_{\perp}^{\top} X B_{\perp}\right)^{-1} z_{k}^{1}$. Computing, the difference $\Delta V:=V\left(z_{k+1}^{1}\right)-V\left(z_{k}^{1}\right)$ along the trajectories of $(65)$ and setting $G:=B_{\perp}^{\top} X B_{\perp}$ and $s_{k}:=G^{-1} z_{k}^{1}$, yields

$$
\begin{aligned}
\Delta V= & \frac{1}{2} z_{k+1}^{1 \top}\left(B_{\perp}^{\top} X B_{\perp}\right)^{-1} z_{k+1}^{1}-\frac{1}{2} z_{k}^{1 \top}\left(B_{\perp}^{\top} X B_{\perp}\right)^{-1} z_{k}^{1} \\
= & \frac{1}{2} s_{k}^{\top} B_{\perp}^{\top} X\left(I_{n}+h A+h \Delta_{A}\right)^{\top} B_{\perp} G^{-1} B_{\perp}^{\top}\left(I_{n}+h A+h \Delta_{A}\right) X B_{\perp} s_{k}-\frac{1}{2} s_{k}^{\top} G s_{k} \\
= & \frac{h}{2} s_{k}^{\top} B_{\perp}^{\top}\left(A X+X A^{\top}+h X A^{\top} B_{\perp} G^{-1} B_{\perp}^{\top} A X\right) B_{\perp} s_{k}+h s_{k}^{\top} B_{\perp}^{\top} \Delta_{A} X B_{\perp} s_{k} \\
& +h^{2} s_{k}^{\top} B_{\perp}^{\top} X A^{\top} B_{\perp} G^{-1} B_{\perp}^{\top} \Delta_{A} X B_{\perp} s_{k}+\frac{h^{2}}{2} s_{k}^{\top} B_{\perp}^{\top} X \Delta_{A}^{\top} B_{\perp} G^{-1} B_{\perp}^{\top} \Delta_{A} X B_{\perp} s_{k} .
\end{aligned}
$$

Making use of the inequality $2 p^{\top} W^{\top} Y q \leq p^{\top} W^{\top} \Psi W p+q^{\top} Y^{\top} \Psi^{-1} Y q$, where $\Psi=\Psi^{\top}>0$, gives us the bounds

$$
\begin{aligned}
s_{k}^{\top} B_{\perp}^{\top} \Delta_{A} X B_{\perp} s_{k} \leq & \frac{1}{2} s_{k}^{\top} B_{\perp}^{\top} \Delta_{A} \Psi_{1} \Delta_{A}^{\top} B_{\perp} s_{k}+\frac{1}{2} s_{k}^{\top} B_{\perp}^{\top} X \Psi_{1}^{-1} X B_{\perp} s_{k} \\
s_{k}^{\top} B_{\perp}^{\top} X A^{\top} B_{\perp} G^{-1} B_{\perp}^{\top} \Delta_{A} X B_{\perp} s_{k} \leq & \frac{1}{2} s_{k}^{\top} B_{\perp}^{\top} X A^{\top} B_{\perp} G^{-1} \Psi_{2} G^{-1} B_{\perp}^{\top} A X B_{\perp} s_{k} \\
& +\frac{1}{2} s_{k}^{\top} B_{\perp}^{\top} X \Delta_{A}^{\top} B_{\perp} \Psi_{2}^{-1} B_{\perp}^{\top} \Delta_{A} X B_{\perp} s_{k} .
\end{aligned}
$$

Setting $\Psi_{1}=\Lambda$ where $\Lambda$ is any positive definite matrix that satisfies Assumption 3, and $\Psi_{2}=G$, then applying the results from Propositions 7 and 8 transforms (66) into

$$
\Delta V \leq-h s_{k}^{\top} B_{\perp}^{\top}\left(a X-\frac{1}{2} I_{n}-\frac{1}{2} X \Lambda^{-1} X-h X \Lambda^{-1} X-\frac{h}{2} X A^{\top} B_{\perp}\left(B_{\perp}^{\top} X B_{\perp}\right)^{-1} B_{\perp}^{\top} A X\right) B_{\perp} s_{k} .
$$

Therefore, $\Delta V<0$ if and only if

$$
B_{\perp}^{\top}\left(a X-\frac{1}{2} I_{n}-\frac{1}{2} X \Lambda^{-1} X-h X \Lambda^{-1} X-\frac{h}{2} X A^{\top} B_{\perp}\left(B_{\perp}^{\top} X B_{\perp}\right)^{-1} B_{\perp}^{\top} A X\right) B_{\perp}>0 .
$$

Notice the resemblance of (70) with (18). In fact, it is easy to see once again that $X \rightarrow P$ as $h \downarrow 0$ where $P$ is a solution of (18). Similarly to the continuous-time case, we will ask for a stronger version of (70). Namely,

$$
\bar{Q}:=\left[\begin{array}{cc}
\bar{Q}_{11} & -\frac{1}{2} B_{\perp}^{\top} A B-\frac{h}{2} B_{\perp}^{\top} X A^{\top} B_{\perp} G^{-1} B_{\perp}^{\top} A B \\
-\frac{1}{2} B^{\top} A^{\top} B_{\perp}-\frac{h}{2} B^{\top} A^{\top} B_{\perp} G^{-1} B_{\perp}^{\top} A X B_{\perp} & \bar{Q}_{22}
\end{array}\right]>0,
$$

where $\bar{Q}_{11}:=B_{\perp}^{\top}\left(a X-I_{n}-\frac{1}{2} X \Lambda^{-1} X-h\left(2 X \Lambda^{-1} X+X A^{\top} B_{\perp}\left(B_{\perp}^{\top} X B_{\perp}\right)^{-1} B_{\perp}^{\top} A X\right)\right) B_{\perp}$ and $\bar{Q}_{22}:=K-\frac{1}{2} B^{\top} \Lambda^{-1} B-h B^{\top}\left(2 \Lambda^{-1}+\frac{3}{2} A^{\top} B_{\perp} G^{-1} B_{\perp}^{\top} A\right) B$. It is also worth to notice that as $h$ decreases to zero, a solution $(X, K)$ of the matrix inequality (71) tends to a solution of the matrix inequality (19). Additionally, in analogy with the continuous-time context, a series of application of the Schur's complement formula gives us the equivalence between the matrix inequality (71) and the following LMI,

$$
\left[\begin{array}{ll}
R_{11} & R_{12} \\
R_{12}^{\top} & R_{22}
\end{array}\right]>0
$$


where,

$$
\begin{aligned}
R_{11}:= & {\left[\begin{array}{ccc}
B_{\perp}^{\top}\left(a X-I_{n}\right) B_{\perp} & -\frac{1}{2} B_{\perp}^{\top} A B & -h B_{\perp}^{\top} X A^{\top} B_{\perp} \\
-\frac{1}{2} B^{\top} A^{\top} B_{\perp} & K & -h B^{\top} A^{\top} B_{\perp} \\
-h B_{\perp}^{\top} A X B_{\perp} & -h B_{\perp}^{\top} A B & 2 h B_{\perp}^{\top} X B_{\perp}
\end{array}\right] } \\
R_{12}:= & {\left[\begin{array}{cccc}
-h B_{\perp}^{\top} X A^{\top} B_{\perp} & 0 & B_{\perp}^{\top} X & 0 \\
0 & -h B^{\top} A^{\top} B_{\perp} & 0 & B^{\top} \\
0 & 0 & 0 & 0
\end{array}\right] } \\
R_{22}:= & {\left[\begin{array}{cccc}
2 h B_{\perp}^{\top} X B_{\perp} & 0 & 0 & 0 \\
0 & h B_{\perp}^{\top} X B_{\perp} & 0 & 0 \\
0 & 0 & \frac{2}{1+2 h} \Lambda & 0 \\
0 & 0 & 0 & \frac{2}{1+2 h} \Lambda
\end{array}\right] . }
\end{aligned}
$$

Assumption 8. Along all this section we will assume that $X$ and $K$ are such that (48), (50) and (72) hold.

The following result is about the conditions in the state for achieving the discrete-time sliding phase $\left(\tilde{\sigma}_{k+1}=\tilde{\sigma}_{k}=0\right.$ for all $k \geq k^{*}$ for some $\left.0<k^{*}<+\infty\right)$.

Lemma 2. Let Assumption 6 hold. The following two statements are equivalent:

1) $\sigma_{k} \in h \gamma \mathbf{M}(0)$ for some $k \in \mathbb{N}$.

2) $\tilde{\sigma}_{k+1}=0$.

In addition, if for some $k_{0} \in \mathbb{N}, \tilde{\sigma}_{k_{0}+1}=0$, then $\tilde{\sigma}_{k_{0}+n}=0$ for all $n \geq 1$, whenever $w_{k}+\eta_{k}^{m} \in \gamma \mathbf{M}(0)$ for all $k \geq k_{0}$.

Proof. The equivalence between 1) and 2) is clear from (64). Namely, $\tilde{\sigma}_{k+1}=0$ is equivalent to $J_{\mathbf{N}}^{h}\left(\sigma_{k}\right)=0$ which in fact is the same as $\sigma_{k} \in(I+h(K+\gamma \mathbf{M}))(0)$. For the second part of the proof we start assuming that for some $k_{0} \in \mathbb{N}, \tilde{\sigma}_{k_{0}+1}=0$. Hence, again from (64) it follows that,

$$
\sigma_{k_{0}+1}=\tilde{\sigma}_{k_{0}+1}+h\left(w_{k_{0}}+\eta_{k_{0}}^{m}\right)=h\left(w_{k_{0}}+\eta_{k_{0}}^{m}\right) \in h \gamma \mathbf{M}(0) .
$$

Therefore, applying the first part of the lemma we obtain $\tilde{\sigma}_{k_{0}+2}=0$. The results follows by induction.

The following result supports the use of the scheme proposed in [1,2].

Corollary 2. Let the matched disturbance $w_{k}+\eta_{k}^{m} \in \gamma \mathbf{M}(0)$ for all $k \geq k^{*}$ for some $0<k^{*}<+\infty$. Then, in the discrete-time sliding phase the control input $u_{k}^{\text {sv }}$ satisfies:

$$
u_{k}^{\mathrm{sv}}=w_{k-1}+\eta_{k-1}^{m} .
$$

Proof. Since in sliding phase $\tilde{\sigma}_{k+1}=\tilde{\sigma}_{k}=0$ it follows from (63) that $u_{k}^{\mathrm{sv}}=-\frac{\sigma_{k}}{h}$ and from (64) we have that $\sigma_{k}=h\left(w_{k-1}+\eta_{k-1}^{m}\right)$ and the result follows.

In words, the input obtained from the implicit scheme (61) compensates for the disturbance with a delay of one step once the discrete-time sliding phase has been reached. Moreover, it is worth to notice that in the discrete-time sliding phase the input $u_{k}^{\mathrm{sv}}$ is independent of the gain $\gamma$, a crucial fact that is experimentally verified in $[25,26]$. This last property becomes fundamental in the application of the control scheme (61) since it helps to drastically reduce the chattering effect of the closed-loop system.

Remark 4. It is worth to mention that the scheme proposed in [1], [2] and stated in (61) for the computation of the control input seems to be connected to the approach of integral sliding modes for the estimation of the disturbance [43]. Indeed, we can see that equation (61c) represents some sort of nominal system from which the control input is obtained instead of using the perturbed system (61b). Moreover, Corollary 2 confirms that, as a consequence of taking the implicit discretization, the obtained controller is automatically compensating the matched disturbance terms with a one-step delay. 
Practical stability of the difference equation (61) is proved by the following theorem.

Theorem 4. Let Assumptions 1-7 hold. Consider the closed-loop system (61) where $X=X^{\top}>0$ and $K=K^{\top}>0$ are such that Assumption 8 holds. In addition, let $L_{c} \subset \mathbb{R}^{n}$ be the compact set

$$
L_{c}:=\left\{\left[\begin{array}{c}
z^{1} \\
\sigma
\end{array}\right] \in \mathbb{R}^{n} \mid \frac{1}{2} z^{1 \top}\left(B_{\perp}^{\top} X B_{\perp}\right)^{-1} z^{1}+\frac{1}{2} \sigma^{\top} \sigma \leq c^{2}\right\} .
$$

Then, for any initial condition $z_{0}=\left[\begin{array}{ll}z_{0}^{1 \top} & \sigma_{0}^{\top}\end{array}\right]^{\top}$ which lies in $L_{c}$ for some $c>0$, there exists $h>0$ small enough and fixed such that for $\gamma>0$ satisfying:

$$
\gamma \varepsilon \geq \beta+W+\left(\sqrt{\bar{\kappa}}+2 h\|K\|^{2}\right) \bar{z},
$$

where $\bar{z}:=2 c^{2} / R$ is an upper bound of $z_{k}$ in $L_{c}$ and $R:=\min \left\{\frac{1}{\lambda_{\max }\left(B_{\perp}^{\top} X B_{\perp}\right)}, 1\right\}$, the discrete-time closed-loop system (61a)-(61d) is semi-globally practically stable. In fact, for any initial condition $z_{0} \in L_{c}$ the trajectories converge to the set $L_{c^{*}}$ where $c>c^{*}:=\sqrt{h \frac{l R}{2 \lambda_{\min }(\hat{Q})}}$ with $l>0$ a constant specified below in the proof.

Proof. Mimicking (41), let us consider the Lyapunov function candidate $V^{k}\left(z^{1}, \sigma\right)=V_{z^{1}}^{k}+V_{\sigma}^{k}$, where $V_{z^{1}}^{k}:=\frac{1}{2} z_{k}^{\top \top}\left(B_{\perp}^{\top} X B_{\perp}\right)^{-1} z_{k}^{1}$ and $V_{\sigma}^{k}:=\frac{1}{2} \sigma_{k}^{\top} \sigma_{k}$. Let $\Delta V=\Delta V_{z^{1}}+\Delta V_{\sigma}$ where $\Delta V_{\sigma}:=V_{\sigma}^{k+1}-V_{\sigma}^{k}$ and $\Delta V_{z^{1}}:=V_{z^{1}}^{k+1}-V_{z^{1}}^{k}$. We split the proof in two parts. The first part consists in finding a proper upper-bound for the difference $\Delta V_{\sigma}$. After this, we continue analyzing the term $\Delta V_{z^{1}}$. Finally we put all terms together and the practical stability follows. Consider the positive definite function $V_{\tilde{\sigma}}^{k}=\frac{1}{2} \tilde{\sigma}_{k}^{\top} \tilde{\sigma}_{k}$ and its respective difference $\Delta V_{\tilde{\sigma}}=V_{\tilde{\sigma}}^{k+1}-V_{\tilde{\sigma}}^{k}$. Then, making use of (61c) and (61d) it follows that,

$$
\begin{aligned}
\Delta V_{\tilde{\sigma}} & =\frac{1}{2} \tilde{\sigma}_{k+1}^{\top} \tilde{\sigma}_{k+1}-\frac{1}{2} \tilde{\sigma}_{k}^{\top} \tilde{\sigma}_{k} \\
& =\frac{1}{2} \tilde{\sigma}_{k+1}^{\top}\left(\tilde{\sigma}_{k+1}-\sigma_{k}\right)-\frac{1}{2} \tilde{\sigma}_{k}^{\top} \tilde{\sigma}_{k}+\frac{1}{2} \tilde{\sigma}_{k+1}^{\top} \sigma_{k} \\
& =\tilde{\sigma}_{k+1}^{\top}\left(\tilde{\sigma}_{k+1}-\sigma_{k}\right)-\frac{1}{2} \tilde{\sigma}_{k}^{\top} \tilde{\sigma}_{k}+\tilde{\sigma}_{k+1}^{\top} \sigma_{k}-\frac{1}{2} \tilde{\sigma}_{k+1}^{\top} \tilde{\sigma}_{k+1} \\
& \leq-h \tilde{\sigma}_{k+1}^{\top}\left(K \tilde{\sigma}_{k+1}+\gamma \zeta_{k+1}\right)+V_{\sigma}^{k}-V_{\tilde{\sigma}}^{k},
\end{aligned}
$$

where $\zeta_{k+1} \in \mathbf{M}\left(\tilde{\sigma}_{k+1}\right)$ and we have used the inequality $2 \tilde{\sigma}_{k+1}^{\top} \sigma_{k} \leq \tilde{\sigma}_{k+1}^{\top} \tilde{\sigma}_{k+1}+\sigma_{k}^{\top} \sigma_{k}$ in the last step. Adding and subtracting the term $V_{\sigma}^{k+1}+V_{\tilde{\sigma}}^{k+1}$ in (76) yields

$$
\Delta V_{\tilde{\sigma}} \leq-h \tilde{\sigma}_{k+1}^{\top} K \tilde{\sigma}_{k+1}-h \gamma \tilde{\sigma}_{k+1}^{\top} \zeta_{k+1}+\frac{1}{2} \sigma_{k+1}^{\top} \sigma_{k+1}-\frac{1}{2} \tilde{\sigma}_{k+1}^{\top} \tilde{\sigma}_{k+1}+\Delta V_{\tilde{\sigma}}-\Delta V_{\sigma},
$$

which, after substitution of (61c) into (61b) leads to,

$$
\begin{aligned}
\Delta V_{\sigma} \leq & -h \tilde{\sigma}_{k+1}^{\top} K \tilde{\sigma}_{k+1}-h \gamma \tilde{\sigma}_{k+1}^{\top} \zeta_{k+1}-\frac{1}{2} \tilde{\sigma}_{k+1}^{\top} \tilde{\sigma}_{k+1} \\
& +\frac{1}{2}\left(\tilde{\sigma}_{k+1}+h\left(w_{k}+\eta_{k}^{m}\right)\right)^{\top}\left(\tilde{\sigma}_{k+1}+h\left(w_{k}+\eta_{k}^{m}\right)\right) \\
= & -h \tilde{\sigma}_{k+1}^{\top} K \tilde{\sigma}_{k+1}-h \gamma \tilde{\sigma}_{k+1}^{\top} \zeta_{k+1}+h \tilde{\sigma}_{k+1}^{\top}\left(w_{k}+\eta_{k}^{m}\right)+h^{2}\left\|w_{k}+\eta_{k}^{m}\right\|^{2} .
\end{aligned}
$$

From (61c) and (61d) it follows that $\tilde{\sigma}_{k+1}=\sigma_{k}-h K \tilde{\sigma}_{k+1}-h \gamma \zeta_{k+1}$, with $\zeta_{k+1} \in \mathbf{M}\left(\tilde{\sigma}_{k+1}\right)$. Then (77) transforms into,

$$
\begin{aligned}
\Delta V_{\sigma} \leq & -h\left(\sigma_{k}-h K \tilde{\sigma}_{k+1}-h \gamma \zeta_{k+1}\right)^{\top} K\left(\sigma_{k}-h K \tilde{\sigma}_{k+1}-h \gamma \zeta_{k+1}\right)-h \gamma \tilde{\sigma}_{k+1}^{\top} \zeta_{k+1} \\
& +h \tilde{\sigma}_{k+1}^{\top}\left(w_{k}+\eta_{k}^{m}\right)+h^{2}\left\|w_{k}+\eta_{k}^{m}\right\|^{2} \\
\leq & -h \sigma_{k}^{\top} K \sigma_{k}+2 h^{2} \sigma_{k}^{\top} K\left(K \tilde{\sigma}_{k+1}+\gamma \zeta_{k+1}\right)-h \gamma \tilde{\sigma}_{k+1}^{\top} \zeta_{k+1}+h \tilde{\sigma}_{k+1}^{\top}\left(w_{k}+\eta_{k}^{m}\right) \\
& +h^{2}\left\|w_{k}+\eta_{k}^{m}\right\|^{2} \\
\leq & -h \sigma_{k}^{\top} K \sigma_{k}-h\left(\gamma \varepsilon-\left\|w_{k}+\eta_{k}^{m}\right\|-2 h\|K\|_{m}^{2}\left\|\sigma_{k}\right\|\right)\left\|\tilde{\sigma}_{k+1}\right\|+2 h^{2} \gamma\|K\|_{m}\left\|\zeta_{k+1}\right\|\left\|\sigma_{k}\right\| \\
& +\frac{h^{2}}{2}\left\|w_{k}+\eta_{k}^{m}\right\|^{2},
\end{aligned}
$$


where we made use of Proposition 6 in the last step. On the other hand, let us recall that $G=$ $\left(B_{\perp}^{\top} X B_{\perp}\right)$ and let us set $s_{k}:=G^{-1} z_{k}^{1}$. Substitution of (61a) into $\Delta V_{z^{1}}$, after some simple algebra, leads to

$$
\begin{aligned}
\Delta V_{z^{1}}= & \frac{1}{2} z_{k+1}^{1 \top} G^{-1} z_{k+1}^{1}-\frac{1}{2} z_{k}^{1 \top} G^{-1} z_{k}^{1} \\
= & \frac{1}{2}\left(B_{\perp}^{\top}\left(I_{n}+h A+h \Delta_{A}\right) X B_{\perp} s_{k}+B_{\perp}^{\top}\left(I_{n}+h A+h \Delta_{A}\right) B \sigma_{k}\right)^{\top} G^{-1}\left(B _ { \perp } ^ { \top } \left(I_{n}+h A\right.\right. \\
& \left.\left.+h \Delta_{A}\right) X B_{\perp} s_{k}+B_{\perp}^{\top}\left(I_{n}+h A+h \Delta_{A}\right) B \sigma_{k}\right)-\frac{1}{2} s_{k}^{\top} G s_{k} \\
= & \frac{1}{2} s_{k}^{\top} B_{\perp}^{\top} X\left(I_{n}+h A+h \Delta_{A}\right)^{\top} B_{\perp} G^{-1} B_{\perp}^{\top}\left(I_{n}+h A+h \Delta_{A}\right) X B_{\perp} s_{k}-\frac{1}{2} s_{k}^{\top} G s_{k} \\
& +s_{k}^{\top} B_{\perp}^{\top} X\left(I_{n}+h A+h \Delta_{A}\right)^{\top} B_{\perp} G^{-1} B_{\perp}^{\top}\left(h A+h \Delta_{A}\right) B \sigma_{k} \\
& +\frac{h^{2}}{2} \sigma_{k}^{\top} B^{\top}\left(A+\Delta_{A}\right)^{\top} B_{\perp} G^{-1} B_{\perp}^{\top}\left(A+\Delta_{A}\right) B \sigma_{k} .
\end{aligned}
$$

Notice that the first two terms in (79) are equal to (66). Then, from (69) it follows that

$$
\begin{aligned}
\Delta V_{z^{1}} \leq & -h s_{k}^{\top} B_{\perp}^{\top}\left(a X-\frac{1}{2} I_{n}-\left(\frac{1}{2}+h\right) X \Lambda^{-1} X-\frac{h}{2} X A^{\top} B_{\perp}\left(B_{\perp}^{\top} X B_{\perp}\right)^{-1} B_{\perp}^{\top} A X\right) B_{\perp} s_{k} \\
& +h s_{k}^{\top} B_{\perp}^{\top} A B \sigma_{k}+h s_{k}^{\top} B_{\perp}^{\top} \Delta_{A} B \sigma_{k}+h^{2} s_{k}^{\top} B_{\perp}^{\top} X A^{\top} B_{\perp} G^{-1} B_{\perp}^{\top} A B \sigma_{k} \\
& +h^{2} s_{k}^{\top} B_{\perp}^{\top} X \Delta_{A}^{\top} B_{\perp} G^{-1} B_{\perp}^{\top} \Delta_{A} B \sigma_{k}+h^{2} s_{k}^{\top} B_{\perp}^{\top} X A^{\top} B_{\perp} G^{-1} B_{\perp}^{\top} \Delta_{A} B \sigma_{k} \\
& +h^{2} s_{k}^{\top} B_{\perp}^{\top} X \Delta_{A}^{\top} B_{\perp} G^{-1} B_{\perp}^{\top} A B \sigma_{k}+\frac{h^{2}}{2} \sigma_{k}^{\top} B^{\top} A^{\top} B_{\perp} G^{-1} B_{\perp}^{\top} A B \sigma_{k} \\
& +\frac{h^{2}}{2} \sigma_{k}^{\top} B^{\top} \Delta_{A}^{\top} B_{\perp} G^{-1} B_{\perp}^{\top} \Delta_{A} B \sigma_{k}+h^{2} \sigma_{k}^{\top} B^{\top} A^{\top} B_{\perp} G^{-1} B_{\perp}^{\top} \Delta_{A} B \sigma_{k} .
\end{aligned}
$$

Applying the inequality $2 p^{\top} U^{\top} \Psi V q \leq p^{\top} U^{\top} \Psi U p+q^{\top} V^{\top} \Psi^{-1} V q$, where $\Psi=\Psi^{\top}>0$, to every cross term in which $\Delta_{A}$ appears in (79), yields the following bounds

$$
\begin{aligned}
s_{k}^{\top} B_{\perp}^{\top} \Delta_{A} B \sigma_{k} \leq & \frac{1}{2} s_{k}^{\top} B_{\perp}^{\top} \Delta_{A} \Psi_{1} \Delta_{A}^{\top} B_{\perp} s_{k}+\frac{1}{2} \sigma_{k}^{\top} B^{\top} \Psi_{1}^{-1} B \sigma_{k} \\
s_{k}^{\top} B_{\perp}^{\top} X \Pi_{1}^{\top} B_{\perp} G^{-1} B_{\perp}^{\top} \Pi_{2} B \sigma_{k} \leq & \frac{1}{2} s_{k}^{\top} B_{\perp}^{\top} X \Pi_{1}^{\top} B_{\perp} G^{-1} \Psi_{2} G^{-1} B_{\perp}^{\top} \Pi_{1} X B_{\perp} s_{k} \\
& +\frac{1}{2} \sigma_{k}^{\top} B^{\top} \Pi_{2}^{\top} B_{\perp} \Psi_{2}^{-1} B_{\perp}^{\top} \Pi_{2} B \sigma_{k} \\
\sigma_{k}^{\top} B^{\top} A^{\top} B_{\perp} G^{-1} B_{\perp}^{\top} \Delta_{A} B \sigma_{k} \leq & \frac{1}{2} \sigma_{k}^{\top} B^{\top} A^{\top} B_{\perp} G^{-1} \Psi_{2} G^{-1} B_{\perp}^{\top} A B \sigma_{k} \\
& +\frac{1}{2} \sigma_{k}^{\top} B^{\top} \Delta_{A}^{\top} B_{\perp} \Psi_{2}^{-1} B_{\perp}^{\top} \Delta_{A} B \sigma_{k},
\end{aligned}
$$

where we set $\Pi_{1}=A$ or $\Pi_{1}=\Delta_{A}$ according to the term in question and similarly for $\Pi_{2}$. Setting $\Psi_{1}=\Lambda$ and $\Psi_{2}=G$, the substitution of previous bounds into (80) gives,

$$
\begin{aligned}
\Delta V_{z^{1}} \leq & -h s_{k}^{\top} B_{\perp}^{\top}\left(a X-\frac{1}{2} I_{n}-\left(\frac{1}{2}+h\right) X \Lambda^{-1} X-\frac{h}{2} X A^{\top} B_{\perp}\left(B_{\perp}^{\top} X B_{\perp}\right)^{-1} B_{\perp}^{\top} A X\right) B_{\perp} s_{k} \\
& +h s_{k}^{\top} B_{\perp}^{\top} A B \sigma_{k}+\frac{h}{2} s_{k}^{\top} B_{\perp}^{\top} \Delta_{A} \Lambda \Delta_{A}^{\top} B_{\perp} s_{k}+\frac{h}{2} \sigma_{k} B^{\top} \Lambda^{-1} B \sigma_{k} \\
& +h^{2} s_{k}^{\top} B_{\perp}^{\top} X A^{\top} B_{\perp} G^{-1} B_{\perp}^{\top} A B \sigma_{k}+h^{2} s_{k}^{\top} B_{\perp}^{\top} X \Delta_{A}^{\top} B_{\perp} G^{-1} B_{\perp}^{\top} \Delta_{A} X B_{\perp} s_{k} \\
& +\frac{h^{2}}{2} s_{k}^{\top} B_{\perp}^{\top} X A^{\top} B_{\perp} G^{-1} B_{\perp}^{\top} A X B_{\perp} s_{k}+\frac{3 h^{2}}{2} \sigma_{k}^{\top} B^{\top} A^{\top} B_{\perp} G^{-1} B_{\perp}^{\top} A B \sigma_{k} \\
& +2 h^{2} \sigma_{k}^{\top} B^{\top} \Delta_{A}^{\top} B_{\perp} G^{-1} B_{\perp}^{\top} \Delta_{A} B \sigma_{k} .
\end{aligned}
$$


Taking into account (51) together with Assumption 3, reduces (81) into

$$
\begin{aligned}
\Delta V_{z^{1}} \leq & -h s_{k}^{\top} B_{\perp}^{\top}\left(a X-I_{n}-\left(\frac{1}{2}+2 h\right) X \Lambda^{-1} X-h X A^{\top} B_{\perp}\left(B_{\perp}^{\top} X B_{\perp}\right)^{-1} B_{\perp}^{\top} A X\right) B_{\perp} s_{k} \\
& +h s_{k}^{\top} B_{\perp}^{\top} A B \sigma_{k}+h^{2} s_{k}^{\top} B_{\perp}^{\top} X A^{\top} B_{\perp} G^{-1} B_{\perp}^{\top} A B \sigma_{k} \\
& +h \sigma_{k} B^{\top}\left(\left(\frac{1}{2}+2 h\right) \Lambda^{-1}+\frac{3}{2} h A^{\top} B_{\perp}\left(B_{\perp}^{\top} X B_{\perp}\right)^{-1} B_{\perp}^{\top} A\right) B \sigma_{k}
\end{aligned}
$$

Addition of (77) and (82) leads to

$$
\begin{aligned}
\Delta V \leq-h z_{k}^{\top} \hat{Q} z_{k}-h\left(\gamma \varepsilon-\left\|w_{k}+\eta_{k}^{m}\right\|-2 h\|K\|_{m}^{2}\left\|\sigma_{k}\right\|\right)\left\|\tilde{\sigma}_{k+1}\right\| & \\
& +2 h^{2} \gamma\|K\|_{m}\left\|\zeta_{k+1}\right\|\left\|\sigma_{k}\right\|+\frac{h^{2}}{2}\left\|w_{k}+\eta_{k}^{m}\right\|^{2}
\end{aligned}
$$

where, $\hat{Q}=\hat{Q}^{\top} \in \mathbb{R}^{n \times n}$ is given as

$$
\hat{Q}:=\left[\begin{array}{cc}
\left(B_{\perp}^{\top} X B_{\perp}\right)^{-1} & 0 \\
0 & I_{m}
\end{array}\right] \bar{Q}\left[\begin{array}{cc}
\left(B_{\perp}^{\top} X B_{\perp}\right)^{-1} & 0 \\
0 & I_{m}
\end{array}\right]>0,
$$

and $\bar{Q}$ is defined in (71). Now, let $L_{c}:=\left\{\left(z_{k}^{1}, \sigma_{k}\right) \in \mathbb{R}^{n} \mid V\left(z_{k}^{1}, \sigma_{k}\right) \leq c^{2}\right\}$ and let $c>0$ be such that $\left(z_{0}^{1}, \sigma_{0}\right) \in L_{c}$ and $\left\|z_{k}\right\|>r$ in the boundary of $L_{c}$ for some $r>0$ fixed. We proceed to show that $L_{c}$ is invariant. To this end, first notice that $\zeta_{k+1} \in \mathbf{M}\left(\tilde{\sigma}_{k+1}\right)$ is bounded in $L_{c}$. Indeed, from (64) and the non-expasiveness property of the resolvent, it follows that $\tilde{\sigma}_{k+1}$ is bounded in $L_{c}$. Additionally, recalling that $\mathbf{M}$ is defined over all $\mathbb{R}^{m}$, it follows that $\mathbf{M}$ is bounded on bounded sets [38, Corollary 12.38] and consequently $\zeta_{k+1} \in \mathbf{M}\left(\tilde{\sigma}_{k+1}\right)$ is bounded in $L_{c}$ by some $\bar{\zeta}>0$. Moreover, it follows from Proposition 9 that in $L_{c},\left\|w_{k}+\eta_{k}^{m}\right\| \leq W+\sqrt{\bar{\kappa}} \bar{z}$. Consequently, for any $\left(z_{k}^{1}, \sigma_{k}\right) \in \operatorname{bd}\left(L_{c}\right)$ we have that

$$
\begin{aligned}
\Delta V \leq & -h \lambda_{\min }(\hat{Q})\left\|z_{k}\right\|^{2}-h\left(\gamma \varepsilon-W-\sqrt{\bar{\kappa}} \bar{z}-2 h\|K\|_{m}^{2} \bar{z}\right)\left\|\tilde{\sigma}_{k+1}\right\| \\
& +2 h^{2} \gamma\|K\|_{m}\|\bar{\zeta}\|\left\|z_{k}\right\|+\frac{h^{2}}{2}\left(W+\sqrt{\bar{\kappa}}\left\|z_{k}\right\|\right)^{2} \\
\leq & -h \lambda_{\min }(\hat{Q})\left\|z_{k}\right\|^{2}-h\left(\gamma \varepsilon-W-\sqrt{\bar{\kappa}} \bar{z}-2 h\|K\|_{m}^{2} \bar{z}\right)\left\|\tilde{\sigma}_{k+1}\right\|+h^{2} l,
\end{aligned}
$$

where $l:=2 \gamma\|K\|_{m}\|\bar{\zeta}\| \bar{z}+\frac{1}{2}(W+\sqrt{\bar{\kappa}} \bar{z})^{2}$. Hence, it follows that for all $z_{k} \in \mathbb{R}^{n}$ such that $\left\|z_{k}\right\|^{2} \geq a h l$ the difference $\Delta V$ is strictly negative for any $a>\frac{1}{\lambda_{\min }(\hat{Q})}$ and in the light of $(75)$ we conclude that the set $L_{c}$ is positively invariant. Finally, noticing that the maximum of $z_{k}$ is reached at the boundary of the set $L_{c}$ and the fact that for any $\bar{c} \in\left(\sqrt{\frac{h a l R}{2}}, c\right)$, where $R=\min \left\{1, \lambda_{\min }\left(B_{\perp}^{\top} X B_{\perp}\right)^{-1}\right\}$ the difference $\Delta V$ is strictly negative at the boundary of the sets $\mathcal{L}_{\bar{c}}$, semi-global practical stability follows.

Remark 5. Practical stability implies the boundary layer approach [44] and in our case we add the prefix semi-global because the disturbance is not uniformly bounded, causing that the gain $\gamma$ will depend on the state for global stability.

Corollary 3. Let all conditions and assumptions of Theorem 4 hold. Let also the gain $\gamma>0$ satisfy:

$$
\gamma \varepsilon \geq \beta+(1+\alpha)(r+W+\sqrt{\bar{\kappa}} \bar{z})+\max \left\{2 h\|K\|^{2} \bar{z}, \frac{(W+\sqrt{\bar{\kappa}} \bar{z})^{2}}{r}\right\},
$$

for some constants $\beta, r>0$ and $\varepsilon>0$ such that $\varepsilon \mathbb{B}_{m} \subset \mathbf{M}(0)$. Then, there exists $k_{0}>0, k_{0}=$ $k_{0}(\alpha, r)$, which is finite and such that the variable $\tilde{\sigma}_{k_{0}}=0$. Moreover, $\tilde{\sigma}_{k}=0$ for all $k \geq k_{0}$, that is, the discrete-time sliding phase is reached in a finite number of steps. 
Proof. From Theorem 4 it follows that for all $k>0$ the state $z_{k}$ is uniformly bounded (since $z_{k} \in L_{c}$ for all $k \geq 0$ ). This boundedness property allows us to analyze the subsystem (64) and to take the disturbance term $w_{k}+\eta_{k}^{m}$ as uniformly bounded. Let us consider first the case were $\left\|\sigma_{k+1}\right\|>h(r+W+\sqrt{\bar{\kappa}} \bar{z})$ for some $k \in \mathbb{N}$ and some $r>0$. Notice that this implies $\left\|\tilde{\sigma}_{k+1}\right\| \geq h r$. Consider the Lyapunov-function candidate $V_{\sigma}=\frac{1}{2} \sigma_{k}^{\top} \sigma_{k}$. From (77) we have that

$$
\begin{aligned}
\Delta V_{\sigma} & \leq-h\left(\gamma \varepsilon-\left\|w_{k}+\eta_{k}^{m}\right\|\right)\left\|\tilde{\sigma}_{k+1}\right\|+h^{2}\left\|w_{k}+\eta_{k}^{m}\right\|^{2} \\
& \leq-h\left(\gamma \varepsilon-(W+\sqrt{\bar{\kappa}} \bar{z})-\frac{(W+\sqrt{\bar{\kappa}} \bar{z})^{2}}{r}\right)\left\|\tilde{\sigma}_{k+1}\right\|
\end{aligned}
$$

Thus, $\Delta V_{\sigma}<0$ whenever $\left\|\sigma_{k+1}\right\|>h(r+W+\sqrt{\bar{\kappa}} \bar{z})$. It follows that $\operatorname{dist}\left(\sigma_{k}, h(r+W+\sqrt{\bar{\kappa}} \bar{z}) \mathbb{B}_{m}\right) \rightarrow$ 0 as $k \rightarrow \infty$. Hence, there exists a finite $k_{0}(\alpha, r)>0$ such that $\left\|\sigma_{k}\right\| \leq(1+\alpha) h(r+W+\sqrt{\bar{\kappa}} \bar{z})$ for all $k \geq k_{0}$, and

$$
\frac{\left\|\sigma_{k}\right\|}{h} \leq(1+\alpha)(r+W+\sqrt{\bar{\kappa}} \bar{z}) \leq \gamma \varepsilon .
$$

Since by assumption $\varepsilon \mathbb{B}_{m} \subset \mathbf{M}(0)$ a direct application of Lemma 2 give us the desired result. On the other hand, if $\left\|\sigma_{k+1}\right\|<h(r+W+\sqrt{\bar{\kappa}} \bar{z})$ we have that

$$
\frac{\left\|\sigma_{k+1}\right\|}{h} \leq r+W+\sqrt{\bar{\kappa}} \bar{z} \leq \gamma \varepsilon
$$

and the proof is complete.

\subsection{Convergence of the discrete-time solutions}

Here we prove that the trajectories of the closed-loop discrete-time system (61) converge to trajectories of the closed-loop continuous-time system (13) as the sampling rate $h>0$ decreases to zero. To this end consider the following piecewise continuous functions

$$
\begin{gathered}
z_{h}^{1}(t):=z_{k}^{1}+\frac{t-t_{k}}{h}\left(z_{k+1}^{1}-z_{k}^{1}\right), \text { for all } t \in\left[t_{k}, t_{k+1}\right] \\
\sigma_{h}(t):=\sigma_{k}+\frac{t-t_{k}}{h}\left(\sigma_{k+1}-\sigma_{k}\right), \text { for all } t \in\left[t_{k}, t_{k+1}\right],
\end{gathered}
$$

together with the step functions

$$
\begin{aligned}
\tilde{\sigma}_{h}^{*}(t) & :=\tilde{\sigma}_{k+1}, \text { for all } t \in\left(t_{k}, t_{k+1}\right] \\
\sigma_{h}^{*}(t) & :=\sigma_{k}, \text { for all } t \in\left(t_{k}, t_{k+1}\right] \\
z_{h}^{1 *}(t) & :=z_{k}, \text { for all } t \in\left(t_{k}, t_{k+1}\right] .
\end{aligned}
$$

From Theorem 4 it follows that for a given initial condition $\left[z_{h}^{1}(0)^{\top}, \sigma_{h}(0)^{\top}\right]^{\top} \in \mathbb{R}^{n}$ the trajectories $z_{h}^{1}$ and $\sigma_{h}$ are maintained, for all time $t>0$, inside a compact set $L_{c}$ for some $c>0$ and hence they are uniformly bounded. Moreover, we have that the derivatives of $z_{h}^{1}$ and $\sigma_{h}$ exist for almost all $t>0$, and satisfy

$$
\begin{aligned}
& \dot{z}_{h}^{1}(t)=\frac{z_{k+1}^{1}-z_{k}^{1}}{h}, \text { for all } t \in\left(t_{k}, t_{k+1}\right) \\
& \dot{\sigma}_{h}(t)=\frac{\sigma_{k+1}-\sigma_{k}}{h}, \text { for all } t \in\left(t_{k}, t_{k+1}\right) .
\end{aligned}
$$

It follows from (61a) and the continuity of $\Delta_{A}$ that $\dot{z}_{h}^{1}$ is uniformly bounded. On the other hand, by (64) we have that

$$
\dot{\sigma}_{h}=\frac{\tilde{\sigma}_{k+1}+h\left(w_{k}+\eta_{k}^{m}\right)-\sigma_{k}}{h}=\frac{J_{\mathbf{N}}^{h}\left(\sigma_{k}\right)-\sigma_{k}}{h}+w_{k}+\eta_{k}^{m}=-\mathcal{N}^{h}\left(\sigma_{k}\right)+w_{k}+\eta_{k}^{m},
$$


where $\mathcal{N}^{h}$ is defined in (63). Thus, from the fact that $\left\|\mathcal{N}^{h}\left(\sigma_{k}\right)\right\| \leq\left\|\operatorname{Proj}_{\mathbf{N}\left(\sigma_{k}\right)}(0)\right\|,[4$, Theorem 2 p. 144] and recalling that $\eta_{k}^{m}=\left(B^{\top} X^{-1} B\right)^{-1} B_{\perp} X^{-1} \Delta_{A}\left(t_{k}, T^{-1} z_{k}\right)$ together with the uniform boundedness of $\Delta_{A}$ and $w_{k}$ (Assumptions 3 and 4 respectively), it follows that $\dot{\sigma}_{h}$ is uniformly bounded too. Hence, we have a pair of equicontinuous sequences of functions $\left\{z_{h}\right\}_{h>0}$ and $\left\{\sigma_{h}\right\}_{h>0}$ and using a similar argument as the one used in the proof of Theorem 1, we get the existence of continuous functions $z^{1}$ and $\sigma$ such that $\left[z_{h}, \sigma_{h}\right] \rightarrow[z, \sigma]$, strongly in $\mathcal{L}_{2}\left([0, T] ; \mathbb{R}^{n}\right)$ and $\left[\dot{z}_{h}, \dot{\sigma}_{h}\right] \rightarrow$ $[\dot{z}, \dot{\sigma}]$ weakly in $\mathcal{L}_{2}\left([0, T] ; \mathbb{R}^{n}\right)$ for any $T>0$. Additionally, we have

$$
\begin{aligned}
\left\|\sigma_{h}-\sigma_{h}^{*}\right\|_{\mathcal{L}_{2}\left([0, T] ; \mathbb{R}^{m}\right)}^{2} & =\sum_{k=0}^{N-1} \int_{t_{k}}^{t_{k+1}}\left(t-t_{k}\right)^{2}\left\|\dot{\sigma}_{h}(t)\right\|^{2} d t \\
& \leq\left. C_{1}^{2} \sum_{k=0}^{N-1} \frac{\left(t-t_{k}\right)^{3}}{3}\right|_{t_{k}} ^{t_{k+1}} \\
& \leq \frac{C_{1}^{2} T h^{2}}{3}
\end{aligned}
$$

where $C_{1}>0$ is an upperbound of $\left\|\dot{\sigma}_{h}\right\|$. Hence $\sigma_{h}^{*} \rightarrow \sigma$ as $h \downarrow 0$. In a similar fashion, we also have $z_{h}^{*} \rightarrow z$ as $h \downarrow 0$. Moreover, as was pointed out above, any solution $X$ of the matrix inequalities (48), (71) converges to a matrix $P$, solution of (5) and (19), as $h$ decreases to zero. Therefore, from (91) and (61) we get:

$$
\begin{aligned}
& \dot{z}_{h}^{1}=B_{\perp}^{\top}\left(A+\Delta_{A}\right) X B_{\perp}\left(B_{\perp}^{\top} X B_{\perp}\right)^{-1} z_{h}^{1 *}+B_{\perp}^{\top}\left(A+\Delta_{A}\right) B \sigma_{h}^{*}, \\
& \rightarrow B_{\perp}^{\top}\left(A+\Delta_{A}\right) P B_{\perp}\left(B_{\perp}^{\top} P B_{\perp}\right)^{-1} z^{1}+B_{\perp}^{\top}\left(A+\Delta_{A}\right) B \sigma=\dot{z}^{1}
\end{aligned}
$$

and

$$
\dot{\sigma}_{h}-w_{h}^{*}-\eta_{h}^{m *} \rightarrow \dot{\sigma}-w-\phi_{m}, \text { as } h \downarrow 0,
$$

both weakly in $\mathcal{L}_{2}\left([0, T] ; \mathbb{R}^{n-m}\right)$ and $\mathcal{L}_{2}\left([0, T] ; \mathbb{R}^{m}\right)$ respectively. Finally, from (92) we have that $-\dot{\sigma}_{h}+w_{h}^{*}+\eta_{h}^{m *}=\mathcal{N}^{h}\left(\sigma_{h}^{*}\right)$ and $J_{\mathbf{N}}^{h}\left(\sigma_{h}^{*}\right) \rightarrow \sigma$ strongly in $\mathcal{L}_{2}\left([0, T] ; \mathbb{R}^{m}\right)$. Indeed,

$$
\begin{aligned}
\left\|\sigma-J_{\mathbf{N}}^{h}\left(\sigma_{h}^{*}\right)\right\| & \leq\left\|\sigma-J_{\mathbf{N}}^{h}(\sigma)\right\|+\left\|J_{\mathbf{N}}^{h}(\sigma)-J_{\mathbf{N}}^{h}\left(\sigma_{h}^{*}\right)\right\| \\
& \leq h\left\|\mathcal{N}^{h}(\sigma)\right\|+\left\|\sigma-\sigma_{h}^{*}\right\| \\
& \leq h\left\|\operatorname{Proj}_{\mathbf{N}(\sigma)}(0)\right\|+\left\|\sigma-\sigma_{h}^{*}\right\|,
\end{aligned}
$$

where we use the non-expasivity of the resolvent. It follows that $J_{\mathbf{N}}^{h}\left(\sigma_{h}^{*}\right) \rightarrow \sigma$ uniformly in $C\left([0, T] ; \mathbb{R}^{m}\right)$ as $h \downarrow 0$, (and consequently, strongly in $\mathcal{L}_{2}\left([0, T] ; \mathbb{R}^{m}\right)$ ). Consequently, using the fact that $\mathcal{N}\left(\sigma_{h}^{*}\right) \in \mathbf{N}\left(J_{\mathbf{N}}^{h}\left(\sigma_{h}^{*}\right)\right)$, where $\mathbf{N}=K+\gamma \mathbf{M}$ [4, Theorem 2 p.144], after the application of Proposition 1 in Section 2 we conclude that the pair $\left(z^{1}, \sigma\right)$ satisfies the differential inclusion (13).

Remark 6. Previous developments reveal that the implicit discretization scheme for the set-valued part of the controller $u_{k}^{\mathrm{sv}}$ makes sense and at the same time allow us to inherit the robustness of the continuous-time closed-loop system.

In the next section we present some numerical examples, showing the robustness of the implemented discrete-time controller as well as the suppression of the chattering effect.

\section{Numerical example}

Consider the following benchmark dynamical system

$$
\dot{x}=\left[\begin{array}{ccccc}
0 & 1 & 0 & 0 & 0 \\
0 & 0 & 1 & 0 & 0 \\
0 & 0 & 0 & 1 & 0 \\
0 & 0 & 0 & 0 & 1 \\
-1 & -2 & 3 & 1 & 2
\end{array}\right] x+\left[\begin{array}{ll}
0 & 0 \\
1 & 0 \\
0 & 0 \\
0 & 1 \\
1 & 1
\end{array}\right] u
$$


$x \in \mathbb{R}^{5}, u \in \mathbb{R}^{2}$, with the parametric uncertainty

$$
\Delta_{A}(t, x)=\left[\begin{array}{ccccc}
0.1 \cos x_{1} & 0.1 & -0.1 & -0.1 & 0 \\
0 & 0.1 \sin x_{2} & 0.2 & 0.3 & -0.4 \\
0.33 & 0.1 & 0 & 0 & -0.1 \sin x_{3} \\
0 & 0 & 0.14 \cos t & 0.2 & 0 \\
1 & 0.4 & 0.1 \sin x_{4} & 0 & 0.1
\end{array}\right]
$$

In addition, we take into account the effects of a matched and bounded external disturbance $w(t)=$ $\left[\begin{array}{ll}2 \sin (t) & 5 \sin (0.63 t)\end{array}\right]^{\top}$. First, we show the continuous-time case with the regularized control law provided by the Yosida approximation of the set-valued map $\mathbf{M}$, and after that, the discrete-time case is exposed. In this example we consider the set-valued map $\mathbf{M}$ as the subdifferential of the infinity norm, i.e., let $f(\sigma)=\|\sigma\|_{\infty}=\max _{i}\left|\sigma_{i}\right|$. Hence,

$$
\begin{aligned}
\mathbf{M}(\sigma) & =\partial f(\sigma):=\left\{\zeta \in \mathbb{R}^{m} \mid f(\eta)-f(\sigma) \geq\langle\zeta, \eta-\sigma\rangle, \text { for all } \eta \in \mathbb{R}^{m}\right\} \\
& =\operatorname{conv}\left\{f^{i}(\sigma) \mid i \in I(\sigma)\right\},
\end{aligned}
$$

where $I(\sigma):=\left\{i \in\{1, \ldots, n\} \mid f^{i}(\sigma)=f(\sigma)\right\}$ is the set of indices where the maximum is achieved [38, Excercise 8.31]. For the continuous-time case we use the regularized controller given by the Yosida approximation to the maximal monotone operator $\mathbf{M}$. Notice that, in the continuous-time case, the selection of the values for reaching the sliding phase will depend of the disturbance terms and therefore there is no suitable selection process. Invoking [7, Example 23.3] we have that $J_{\partial f}^{\mu}=$ $\operatorname{Prox}_{\mu f}$, where Prox ${ }_{\mu f}$ refers to the proximal map of the function $\mu f$ defined in Section 2. In order to compute the Yosida approximation first notice that the Moreau's decomposition Theorem [7, Theorem 14.3] gives us:

$$
\mathcal{M}^{\mu}(\sigma)=\frac{1}{\mu}\left(I-J_{\mathbf{M}}^{\mu}\right)(\sigma)=\operatorname{Prox}_{f^{\star} / \mu}\left(\frac{\sigma}{\mu}\right) .
$$

So, we proceed to compute the conjugate function $f^{\star}(\sigma):=\sup _{x \in \mathbb{R}^{m}}\{\langle x, \sigma\rangle-f(x)\}$. To this end, let us first consider the case when $\sigma$ is such that $\sum_{i}\left|\sigma_{i}\right| \leq 1$. Then we have:

$$
\begin{aligned}
0 & =\langle 0, \sigma\rangle-f(0) \leq f^{\star}(\sigma)=\sup _{x \in \mathbb{R}^{m}}\left\{\langle x, \sigma\rangle-\|x\|_{\infty}\right\} \\
& \leq \sup _{x \in \mathbb{R}^{m}}\left\{\sum_{i=1}^{m}\left|\sigma_{i}\right|\left|x_{i}\right|-\|x\|_{\infty}\right\} \\
& \leq \sup _{x \in \mathbb{R}^{m}}\left\{\|x\|_{\infty}\left(\sum_{i=1}^{m}\left|\sigma_{i}\right|-1\right)\right\}=0 .
\end{aligned}
$$

Hence $f^{\star}(\sigma)=0$ whenever $\|\sigma\|_{1} \leq 1$. On the other hand, consider the case where $\sum_{i}\left|\sigma_{i}\right|>1$. In this case we have:

$$
\begin{aligned}
f^{\star}(\sigma) & =\sup _{x \in \mathbb{R}^{m}}\left\{\langle x, \sigma\rangle-\|x\|_{\infty}\right\} \\
& \geq \sup _{b \in \mathbb{R}_{+}}\left\{\sum_{i=1}^{m} \sigma_{i} b \operatorname{sign}\left(\sigma_{i}\right)\|\sigma\|_{\infty}-b\left\|\left[\begin{array}{c}
\operatorname{sign}\left(\sigma_{1}\right)\|\sigma\|_{\infty} \\
\vdots \\
\operatorname{sign}\left(\sigma_{m}\right)\|\sigma\|_{\infty}
\end{array}\right]\right\|_{\infty}\right\} \\
& =\sup _{b \in \mathbb{R}_{+}}\left\{b\|\sigma\|_{\infty}\left(\sum_{i=1}^{m}\left|\sigma_{i}\right|-1\right)\right\}=+\infty .
\end{aligned}
$$

It follows that $f^{\star}(\sigma)=\Psi_{\mathbb{B}_{m}^{1}}(\sigma)$, where $\mathbb{B}_{m}^{1}:=\left\{x \in \mathbb{R}^{m} \mid\|x\|_{1} \leq 1\right\}$ and the function $\Psi_{C}$ denotes the indicator function of the set $C$. Therefore,

$$
\mathcal{M}^{\mu}(\sigma)=\operatorname{Prox}_{\Psi_{\mathbb{B}_{m}^{1}}}\left(\frac{\sigma}{\mu}\right)=\operatorname{Proj}_{\mathbb{B}_{m}^{1}}\left(\frac{\sigma}{\mu}\right) .
$$


The next step consists in the computation of the sliding surface $C$. Following the steps described in Section 3 we have that $C=\left(B^{\top} P^{-1} B\right) B^{\top} P^{-1}$ where $P=P^{\top}>0$ is a solution of (5), (20). Thus, using the software package CVX [23], together with the solver SeDuMi [40] to solve the LMIs (5) and (20) yields,

$$
P=\left[\begin{array}{ccccc}
2.3075 & -3.3999 & -1.4020 & 2.5063 & -2.0431 \\
-3.3999 & 18.3866 & 1.4443 & -9.7181 & 9.8744 \\
-1.4020 & 1.4443 & 13.8392 & -19.8470 & -9.7614 \\
2.5063 & -9.7181 & -19.8470 & 70.0849 & 38.7141 \\
-2.0431 & 9.8744 & -9.7614 & 38.7141 & 38.7003
\end{array}\right]
$$

together with

$$
K=\left[\begin{array}{cc}
14.6386 & -2.411 \\
-2.4111 & 14.2337
\end{array}\right]
$$

and it follows that,

$$
C=\left[\begin{array}{ccccc}
1.5052 & 0.9790 & 0.0350 & -0.0210 & 0.0210 \\
-0.0019 & -1.7935 & 0.3140 & -0.7935 & 1.7935
\end{array}\right]
$$

Figure 1 shows the trajectories, the sliding variable and the control input of the closed-loop system (93) with regularized control input $u=u^{\text {nom }}-K \sigma-\gamma(z) \mathcal{M}^{\mu}(\sigma)$, taking $\mu=0.001, a=1.4$ and the initial condition $x(0)=\left[\begin{array}{lllll}1 & -1 & 1 & 0 & -1\end{array}\right]^{\top}$. The simulations were carried up in Matlab using a Dormand-Prince solver (ode45) with variable time-step and relative tolerance of $10^{-6}$. Also it is worth to mention that there is no chattering present neither in the input nor in the output $\sigma$, since the control input is Lipschitz continuous, see (63), and well-posed over all $\mathbb{R}^{m}$, see Figure 1.
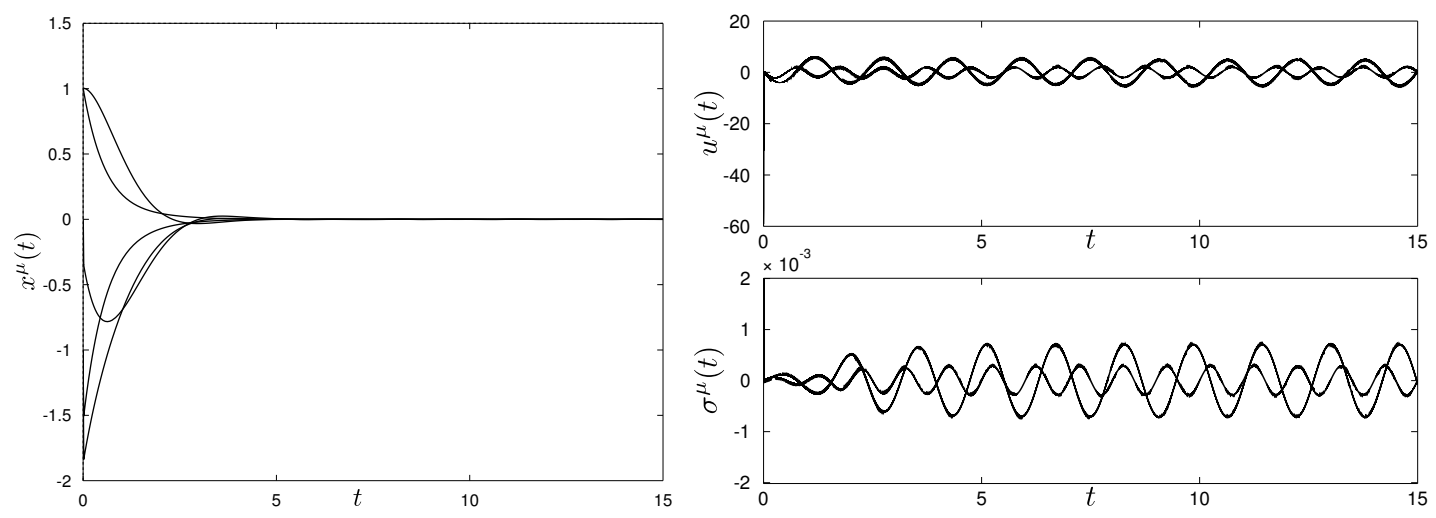

Figure 1: Time evolution of the control input $u=u^{\text {nom }}-K \sigma-\gamma(z) \mathcal{M}^{\mu}(\sigma)$ and the corresponding system trajectories and sliding variable with $\mu=0.001$.

For the discrete-time setting, we simulate the continuous-time plant with a ZOH sampling mechanism and we implement the discrete-time controller exposed in Section 4.3. We use the set-valued maximal monotone map $\mathbf{M}$ defined in (95). In this context, instead of computing the Yosida approximation of $\mathbf{N}=K+\gamma \mathbf{M}$, we introduce another way of computing the control input $u^{\mathrm{sv}}$ from the Yosida approximation of the set-valued map M. From (61c)-(61d) it follows that $\left(I_{n}+h K\right) \tilde{\sigma}_{k+1}-\sigma_{k} \in$ $-h \gamma \mathbf{M}\left(\tilde{\sigma}_{k+1}\right)$ or equivalently,

$$
\begin{aligned}
\theta \sigma_{k}-\theta\left(I_{n}+h K\right) \tilde{\sigma}_{k+1} & \in \theta h \gamma \mathbf{M}\left(\tilde{\sigma}_{k+1}\right) \\
& \Uparrow \\
\theta \sigma_{k}+\left(I_{n}-\theta I_{n}-\theta h K\right) \tilde{\sigma}_{k+1} & \in(I+\theta h \gamma \mathbf{M})\left(\tilde{\sigma}_{k+1}\right) \\
& \mathbb{} \\
\tilde{\sigma}_{k+1} & =J_{\mathbf{M}}^{\theta h \gamma}\left(\theta \sigma_{k}+\left(I_{n}-\theta\left(I_{n}+h K\right)\right) \tilde{\sigma}_{k+1}\right) .
\end{aligned}
$$


We claim that the right-hand side of (96) is a contraction for $\theta>0$ sufficiently small. Indeed, recalling that the resolvent $J_{\mathbf{M}}^{\mu}$ is non-expansive for any $\mu>0$ it follows that:

$$
\begin{aligned}
\left\|J_{\mathbf{M}}^{\theta h \gamma}\left(\theta \sigma_{k}+\left(I_{n}-\theta\left(I_{n}+h K\right)\right) \tilde{\sigma}_{k+1}^{1}\right)-J_{\mathbf{M}}^{\theta h \gamma}\left(\theta \sigma_{k}+\left(I_{n}-\theta\left(I_{n}+h K\right)\right) \tilde{\sigma}_{k+1}^{2}\right)\right\| \\
\leq\left\|I_{n}-\theta\left(I_{n}+h K\right)\right\|_{m}\left\|\tilde{\sigma}_{k+1}^{1}-\tilde{\sigma}_{k+1}^{2}\right\| .
\end{aligned}
$$

Hence, taking $\theta>0$ small enough we have that $\left\|I_{n}-\theta\left(I_{n}+h K\right)\right\|_{m}<1$ and then $J_{\mathbf{M}}^{\theta h \gamma}$ is a contraction. Consequenlty, the successive approximations method can be applied in order to find the fixed point $\tilde{\sigma}_{k+1}$ of (96) and the control input $u_{k}^{\mathrm{sv}}$ at each sampling instant. We set three different sampling periods, $h \in\{50 \mathrm{~ms}, 5 \mathrm{~ms}, 0.5 \mathrm{~ms}\}, a=1.4$ and $x_{0}=\left[\begin{array}{lllll}1 & -1 & 1 & 0 & -1\end{array}\right]^{\top}$ as before. In the three cases we solve (48), (50) and (72) and we obtain the following sliding surfaces $S_{h}, h \in\{50 \mathrm{~ms}, 5 \mathrm{~ms}, 0.5 \mathrm{~ms}\}$ :

$$
\begin{aligned}
S_{h_{1}} & =\left[\begin{array}{lllll}
1.4759 & 0.9867 & 0.0042 & -0.0133 & 0.0133 \\
0.1065 & -1.6527 & 0.6364 & -0.6527 & 1.6527
\end{array}\right] \\
S_{h_{2}} & =\left[\begin{array}{ccccc}
1.4733 & 0.9912 & 0.0266 & -0.0088 & 0.0088 \\
0.0317 & -1.7821 & 0.3248 & -0.7821 & 1.7821
\end{array}\right] \\
S_{h_{3}} & =\left[\begin{array}{lllll}
1.4701 & 0.9977 & 0.0332 & -0.0023 & 0.0023 \\
0.0280 & -1.7837 & 0.3083 & -0.7837 & 1.7837
\end{array}\right] .
\end{aligned}
$$

For the simulation of the system, we use the same Matlab configuration setting as in the previous case. Figures 2-3 show the evolution of the trajectories of the closed-loop system (93) with a control scheme dictated by (61), as well as the evolution in time of the sliding variable and the control input. The subindices in the labels of the plots indicate the sampling time $h$ for the current variable. Notice that in all the three cases there is no chattering at all, neither in the input nor in the output, c.f. Figure 4. It is noteworthy that the control compensates for the disturbance as stated in Corollary 2 .
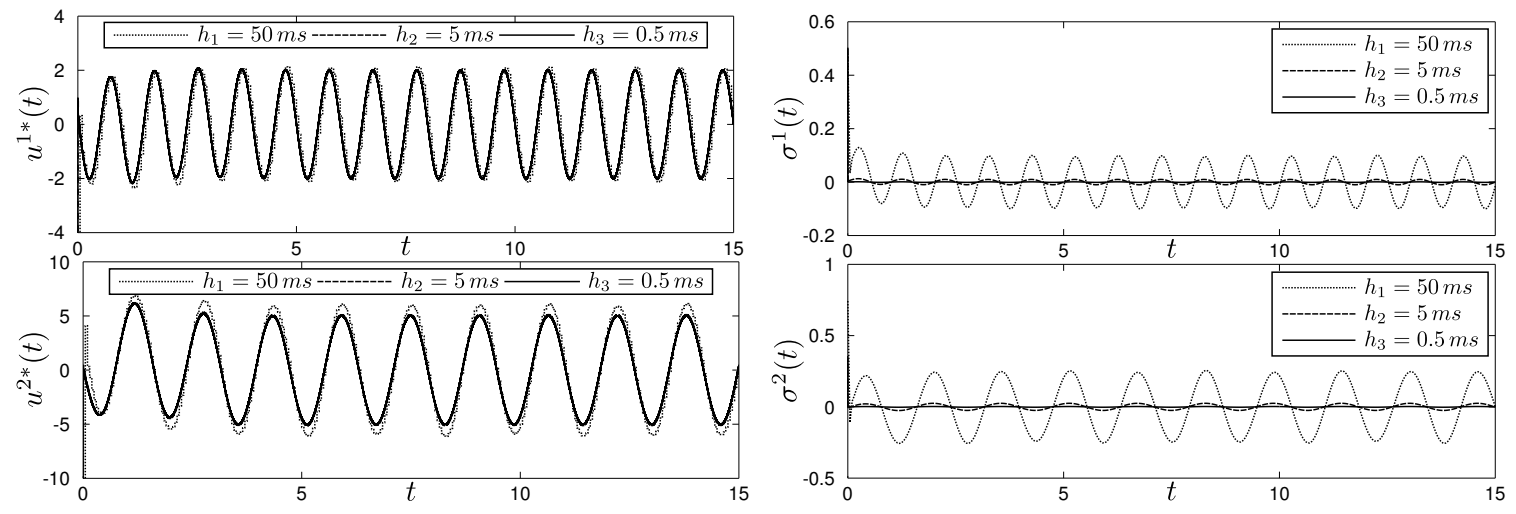

Figure 2: Time evolution of the control input $u_{k}=u_{k}^{\text {nom }}+u_{k}^{\text {sv }}$ (left) and the associated sliding variable (right), for the sampling times $h \in\{50 \mathrm{~ms}, 5 \mathrm{~ms}, 0.5 \mathrm{~ms}\}$.

Finally, Figure 4 shows the plots of the control input, sliding variable and system trajectories of the closed-loop system (93) when the conventional unit vector control is applied using an explicit discretization for the set-valued part of the controller, that is, $u\left(t_{k}\right)=u^{\mathrm{nom}}\left(t_{k}\right)-K \sigma\left(t_{k}\right)-\gamma \frac{\sigma\left(t_{k}\right)}{\left\|\sigma\left(t_{k}\right)\right\|+0.001}$, on $\left[t_{k}, t_{k+1}\right)$, with sampling time $h=5 \mathrm{~ms}$. Notice that, when we regularize the control input in the conventional way there is no selection procedure which at the end is translated into the appearance of chattering in the system. Numerical chattering (i.e., the chattering due to the time-discretization) is known to be intrinsic to explicit discretizations $[21,22,26]$. 


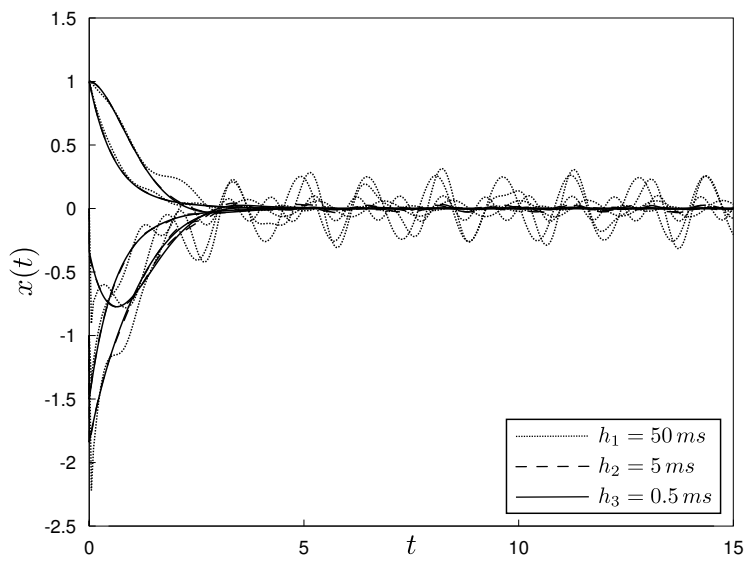

Figure 3: Time evolution of the piecewise linear trajectories $x(t)$ of the discrete-time system $(61)$ for the sampling times $h \in\{50 \mathrm{~ms}, 5 \mathrm{~ms}, 0.5 \mathrm{~ms}\}$.
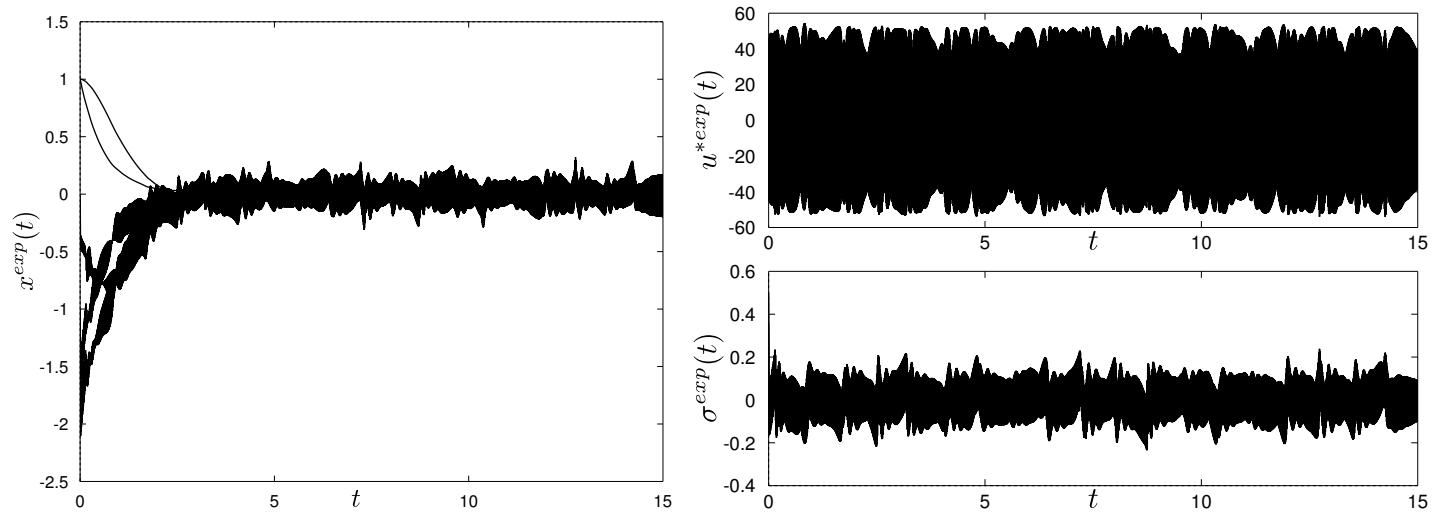

Figure 4: Time evolution of the control input $u=u^{\text {nom }}-K \sigma-\gamma \sigma /(\|\sigma\|+0.001)$ and the corresponding system trajectories and sliding variable with a sampling step $h=5 \mathrm{~ms}$. 


\section{Concluding remarks}

In this work we present a family of set-valued sliding mode controllers making use of the so-called maximal monotone operators. The proposed methodology has the advantage of embracing the two main approaches existents in the literature of design of sliding mode controllers, namely, the unit vector control and the componentwise control among others. Additionally the scheme proposed allows us to deal with the multivariable case without any modification and provides a unique and wellposed way of regularization of the set-valued controller through the use of the Yosida approximation. All along the article we deal with parametric and matched external disturbances. A study for both the continuous and discrete-time cases, was presented. In the continuous-time case it was shown that the proposed set-valued controller is well-posed even in the case when the right-hand side is not maximal monotone. Moreover, the convergence of the trajectories through the use of the Yosida approximation was established. On the other hand, the implementation of the kind of controllers obtained from the continuous-time setting was analyzed. It was shown that the use of the implicit discretization for the set-valued part of the controller is well-posed, and allows us to make a selection for the values of the designed controller that will compensate the disturbances in a unique fashion. The advantage of making a selection rather than switching is translated into the suppression of the chattering effect, confirming previous analytical and experimental results obtained in a less general framework not encompassing parametric uncertainties.

\section{References}

[1] V. Acary and B. Brogliato. Implicit Euler numerical scheme and chattering-free implementation of sliding mode systems. Systems \&3 Control Letters, 59(5):284-293, 2010.

[2] V. Acary, B. Brogliato, and Y. V. Orlov. Chattering-free digital sliding-mode control with state observer and disturbance rejection. Automatic Control, IEEE Transactions on, 57(5): 1087-1101, 2012.

[3] S. Adly, H. Attouch, and A. Cabot. Finite time stabilization of nonlinear oscillators subject to dry friction. In P. Alart, O. Maisonneuve, and R. T. Rockafellar, editors, Progresses in Nonsmooth Mechanics and Analysis, chapter 24. Springer, Dordrecht.

[4] J. Aubin and A. Cellina. Differential Inclusions: Set-Valued Maps and Viability Theory. Grundlehren der mathematischen Wissenschaften. Springer Berlin Heidelberg, 1984.

[5] B. Baji and A. Cabot. An inertial proximal algorithm with dry friction: finite convergence results. Set-Valued Analysis, 14(1):1-23, 2006.

[6] J. Bastien. Convergence order of implicit Euler numerical scheme for maximal monotone differential inclusions. Zeitschrift für angewandte Mathematik und Physik, 64(4):955-966, 2013.

[7] H. Bauschke and P. Combettes. Convex Analysis and Monotone Operator Theory in Hilbert Spaces. CMS Books in Mathematics. Springer New York, 2011.

[8] S. Boyd, L. Ghaoui, E. Feron, and V. Balakrishnan. Linear Matrix Inequalities in System and Control Theory. Studies in Applied Mathematics. Society for Industrial and Applied Mathematics (SIAM, 3600 Market Street, Floor 6, Philadelphia, PA 19104), 1994.

[9] H. Brézis. Opérateurs Maximaux Monotones et Semi-groupes de Contractions dans les Espaces de Hilbert. Number 5 in North-Holland mathematics studies. North-Holland Publishing Company, 1973.

[10] B. Brogliato and D. Goeleven. Well-posedness, stability and invariance results for a class of multivalued Lur'e dynamical systems. Nonlinear Analysis: Theory, Methods and Applications, 74(1):195 - 212, 2011. 
[11] B. Brogliato, A. Daniilidis, C. Lemaréchal, and V. Acary. On the equivalence between complementarity systems, projected systems and differential inclusions. Systems and Control Letters, $55(1): 45-51,2006$.

[12] M. Camlibel and J. Schumacher. Linear passive systems and maximal monotone mappings. Mathematical Programming, Ser. B:1-24, 2015.

[13] C. Castaing, T. X. D. Hā, and M. Valadier. Evolution equations governed by the sweeping process. Set-Valued Analysis, 1(2):109-139, 1993.

[14] F. Castaños and L. Fridman. Analysis and design of integral sliding manifolds for systems with unmatched perturbations. Automatic Control, IEEE Transactions on, 51(5):853-858, 2006.

[15] A. Cellina and M. Marchi. Non-convex perturbations of maximal monotone differential inclusions. Israel Journal of Mathematics, 46(1):1-11, 1983.

[16] H. H. Choi. A new method for variable structure control system design: A linear matrix inequality approach. Automatica, 33(11):2089-2092, 1997.

[17] H. H. Choi. An LMI-based switching surface design method for a class of mismatched uncertain systems. Automatic Control, IEEE Transactions on, 48(9):1634-1638, 2003.

[18] J. Cortés. Discontinuous dynamical systems: A tutorial on solutions, nonsmooth analysis and stability. Control Systems Magazine, IEEE, 28(3):36-73, 2008.

[19] J. F. Edmond and L. Thibault. BV solutions of nonconvex sweeping process differential inclusion with perturbation. Journal of Differential Equations, 226(1):135-179, 2006.

[20] A. Filippov and F. Arscott. Differential Equations with Discontinuous Righthand Sides: Control Systems. Mathematics and its Applications. Springer, 1988.

[21] Z. Galias and X. Yu. Euler's discretization of single input sliding-mode control systems. Automatic Control, IEEE Transactions on, 52(9):1726-1730, 2007.

[22] Z. Galias and X. Yu. Analysis of zero-order holder discretization of two-dimensional slidingmode control systems. IEEE Transactions on Circuits and Systems, 55(12):1269-1273, 2008.

[23] M. Grant and S. Boyd. CVX: Matlab software for disciplined convex programming, version 2.1. http://cvx.com/cvx, Mar. 2014.

[24] O. Huber, V. Acary, and B. Brogliato. Lyapunov stability and performance analysis of the implicit discrete sliding mode control. Automatic Control, IEEE Transactions on, 2016. doi: 10.1109/TAC.2015.2506991.

[25] O. Huber, V. Acary, B. Brogliato, and F. Plestan. Discrete-time twisting controller without numerical chattering: analysis and experimental results with an implicit method. Control Engineering Practice, 46:129-141, 2016.

[26] O. Huber, B. Brogliato, V. Acary, A. Boubakir, F. Plestan, and B. Wang. Experimental results on implicit and explicit time-discretization of equivalent-control-based sliding mode control. In L. Fridman, J.-P. Barbot, and F. Plestan, editors, Recent Trends in Sliding Mode Control, pages 207-236. IET, 2016.

[27] R. E. Kalman, Y. C. Ho, and K. S. Narendra. Controllability of Linear Dynamical Systems. In J. P. LaSalle and J. B. Diaz, editors, Contribution to Differential Equations, volume 1. Interscience, New York, 1963.

[28] A. N. Kolmogorov and S. V. Fomin. Elements of the Theory of Funtions and Functional Analysis, volume 1 and 2. Graylock Press, New York, 1957. 
[29] A. J. Kurdila and M. Zabarankin. Convex Functional Analysis. Systems \& Control: Foundations \& Applications. Birkhäuser Verlag, Germany, 2005.

[30] A. Levant. Higher-order sliding modes, differentiation and output-feedback control. International journal of Control, 76(9-10):924-941, 2003.

[31] F. A. Miranda-Villatoro and F. Castaños. Robust output regulation of variable structure systems with multivalued controls. In Variable Structure Systems (VSS), 2014 13th International Workshop on, pages 1-6, June 2014. doi: 10.1109/VSS.2014.6881118.

[32] F. A. Miranda-Villatoro and F. Castaños. Robust output regulation of strongly linear passive systems with multivalued maximally monotone controls. Automatic Control, IEEE Transactions on, 2016. doi: 10.1109/TAC.2016.2544926.

[33] J.-S. Pang and D. Stewart. Differential variational inequalities. Mathematical Programming, 113(2):345-424, 2008.

[34] W. Perruquetti and J. Barbot, editors. Sliding Mode Control In Engineering. Automation and Control Engineering. CRC Press, 2002.

[35] J. Peypouquet and S. Sorin. Evolution equations for maximal monotone operators: asymptotic analysis in continuous and discrete time. Journal of Convex Analysis, 17(3 \& 4):1113-1163, 2010.

[36] A. Polyakov and A. Poznyak. Invariant ellipsoid method for minimization of unmatched disturbances effects in sliding mode control. Automatica, 47(7):1450-1454, 2011.

[37] R. T. Rockafellar. Convex Analysis. Convex Analysis. Princeton University Press, 1970.

[38] R. T. Rockafellar and R. Wets. Variational Analysis. Die Grundlehren der mathematischen Wissenschaften in Einzeldarstellungen. Springer, 2009.

[39] Y. Shtessel, C. Edwards, L. Fridman, and A. Levant. Sliding Mode Control and Observation. Birkhäuser, 2014.

[40] J. F. Sturm. Using SeDuMi 1.02, a MATLAB toolbox for optimization over symmetric cones. Optimization methods and software, 11(1-4):625-653, 1999.

[41] V. Utkin. Sliding Modes in Control and Optimization. Communications and Control Engineering. Springer-Verlag, 1992.

[42] V. Utkin and A. Poznyak. Adaptive sliding mode control. In B. Bandyopadhyay, S. Janardhanan, and S. Spurgeon, editors, Advances in Sliding Mode Control: Concept, Theory and Implementation, chapter 2, pages 21-54. Springer-Verlag, Berlin, 2013.

[43] V. Utkin and J. Shi. Integral sliding mode in systems operating under uncertainty conditions. In Decision and Control, 1996. Proceedings of the 35th IEEE Conference on, volume 4, pages 4591-4596. IEEE, 1996.

[44] V. Utkin, J. Guldner, and J. Shi. Sliding Mode Control in Electro-Mechanical Systems, volume 34. CRC press, 2009.

[45] B. Wang, B. Brogliato, V. Acary, A. Boubakir, and F. Plestan. Experimental comparisons between implicit and explicit implementations of discrete-time sliding mode controllers: Toward input and output chattering suppression. Control Systems Technology, IEEE Transactions on, 23(5):2071-2075, Sept 2015.

[46] K. D. Young, V. I. Utkin, and U. Ozguner. A control engineer's guide to sliding mode control. IEEE transactions on control systems technology, 7(3):328-342, 1999. 